\title{
A logical approach to fuzzy truth hedges ${ }^{\text {th }}$
}

\author{
Francesc Esteva, Lluís Godo, Carles Noguera \\ Artificial Intelligence Research Institute (IIIA - CSIC) \\ Campus de la Universitat Autònoma de Barcelona s/n, 08193 Bellaterra, Catalonia, Spain \\ \{esteva,godo, cnoguera\} @iiia.csic.es
}

\begin{abstract}
The starting point of this paper are the works of Hájek and Vychodil on the axiomatization of truthstressing and -depressing hedges as expansions of Hájek's BL logic by new unary connectives. They showed that their logics are chain-complete, but standard completeness was only proved for the expansions over Gödel logic. We propose weaker axiomatizations over an arbitrary core fuzzy logic which have two main advantages: (i) they preserve the standard completeness properties of the original logic and (ii) any subdiagonal (resp. superdiagonal) non-decreasing function on $[0,1]$ preserving 0 and 1 is a sound interpretation of the truth-stresser (resp. depresser) connectives. Hence, these logics accommodate most of the truth hedge functions used in the literature about of fuzzy logic in a broader sense.
\end{abstract}

Keywords: Mathematical Fuzzy Logic, Standard completeness, Truth hedges, T-norm based logics.

\section{Introduction}

In pragmatics, hedges are linguistic terms used to mitigate the impact of an utterance (see e.g. [19]). Their usage in fuzzy logic goes back to Lakoff [28], where they can be not only mitigating but also strengthening modifiers. As pointed out in [29], Lakoff was actually concerned with the logical properties of words and phrases like rather, largely, very, in their ability "to make things fuzzier or less fuzzy". In accordance with Lakoff's main concern, however, the term hedge has later been defined [6] as "a particle, word or phrase that modifies the degree of membership of a predicate or a noun phrase in a set; it says of that membership that it is partial or true only in certain respects, or that it is more true and complete than perhaps might be expected". This definition encompasses hedges in the sense of both stressers or depressers depending on whether they strengthen or soften the impact or the meaning of an utterance. ${ }^{1}$ Typical examples are adjectives as in:

They lost a terrible amount of money (stresser),

They lost an insignificant amount of money (depresser),

\footnotetext{
The investigation presented in this paper started with some preliminary results in the conference paper [17], that later were also collected in the Handbook contribution [15, Section 3], and is now presented in its final and complete form.

${ }^{1}$ This terminology is customary in fuzzy logic papers; in other sources, like [19] stressers are called 'intensifiers', while 'hedge' only refers to depressers.
} 
or adverbs in:

Messi is definitely a better player than Maradona ever was (stresser), Messi is slightly a better player than Maradona ever was (depresser).

Clauses can act as hedges too, for example:

I think you should reinstall the operating system of your computer, I know what I'm talking about (stresser),

I'm not an expert, but I think you should reinstall the operating system of your computer (depresser).

Other clauses acting as hedges are those which directly refer to the truth of some sentence like it is very true that, it is quite true that, it is more or less true that, it is slightly true that, etc. Any sentence with a hedge, of any grammatical category, can be translated into one using clauses of the latter kind. In this formulation they have been represented in fuzzy logic systems (in broad sense) as functions from the set of truth values (typically the real unit interval) into itself that modify the meaning of a proposition by being applied to the membership function of the fuzzy set underlying the proposition (see [39]). More specifically, in the setting of mathematical fuzzy logic, Hájek proposes in a series of papers $[23,22,21]$ to understand them as truth functions of new unary connectives, a kind of modal modifiers or truth modifiers, called truth-stressing or truth-depressing hedges depending on whether they reinforce or weaken the meaning of the proposition they are applied to. The intuitive mathematical interpretation of a truth-stressing (resp. depressing) hedge on a chain of truth-values is a subdiagonal (resp. superdiagonal) non-decreasing function preserving 0 and 1 . The class of such functions will be called hedge functions from now on.

This paper builds upon previous works, mainly those by Hájek [22, 21] and Vychodil [38], on the axiomatization of truth-stressing (resp. depressing) hedges as expansions of BL logic (and of some of their prominent extensions, like Eukasiewicz or Gödel logics) by a new unary connective $v t$, for very true, and another one st, for slightly true, respectively. The logics they define are shown to be algebraizable and to enjoy completeness with respect to the classes of chains of their corresponding varieties, however not any BL-chain expanded with hedge functions are models of them, or in other words, belong to the corresponding varieties. Moreover, the defined logics are not proved to enjoy standard completeness in general, except for the case of logics over Gödel logic. One of the main reasons for both problems is the presence in the axiomatizations of the well-known modal axiom $\mathrm{K}$ for the $v t$ connective,

$$
v t(\varphi \rightarrow \psi) \rightarrow(v t \varphi \rightarrow v t \psi),
$$

which puts quite a lot of constraints on the hedges to be models of these logics with no natural algebraic interpretation.

Particular classes of truth-stressers have been also addressed in the literature. For instance, the well-known projection operator $\triangle$ (introduced independently by Monteiro in the context of intuitionistic logic [31] and by Baaz in the context of Gödel-Dummett logics [1]) is a limit case of a truth-stresser since, over a chain, it maps 1 to 1 and all the other elements to 0 , and the intuitive interpretation would be it is definitely true that.

In this paper we propose weaker axiomatizations over any core fuzzy logic for both the truthstressing and -depressing connectives not imposing any constraint on hedges other than the ones we have mentioned above, and for which we can prove standard completeness. 
The paper is structured as follows. After this introduction, Section 2 introduces the previous axiomatic approaches to hedges in fuzzy logic and provides the necessary logical and algebraic preliminaries that are used in the rest of the paper. In Section 3 we propose a general axiomatization for truth-stressers and present its main properties, including standard completeness results. Section 4 proves several versions of the deduction theorem for some logics with truth-stressers and, as a consequence, we obtain that their corresponding algebraic semantics is a variety. Sections 5 and 6 perform an analogous investigation for logics with truth-depressing hedges. In Section 7 we consider some further topics, namely the combination of stressers and depressers, the usage of hedges in the presence of truth-constants, and first-order logics with hedges. Finally, in Section 8 we discuss alternative approaches in the literature.

\section{Preliminaries}

\subsection{Hájek and Vychodil axiomatizations}

As mentioned in the introduction, there are two main references when talking about the formalization of truth hedges within the framework of mathematical fuzzy logic. The first one is Hájek's paper [21] where he axiomatizes over BL a logic for the hedge very true. The second one is the paper by Vychodil [38] where he extends Hájek's analysis to truth-depressing hedges. In the rest of this section we overview these and some related logics and compare them with our proposal.

Hájek defines the logic $\mathrm{BL}_{v t}$ as the expansion of $\mathrm{BL}$ with a new connective $v t$ and the following axioms

$$
\begin{aligned}
& (\mathrm{VT} 1) v t \varphi \rightarrow \varphi, \\
& (\mathrm{VT} 2) v t(\varphi \rightarrow \psi) \rightarrow(v t \varphi \rightarrow v t \psi), \\
& (\mathrm{VT} 3) v t(\varphi \vee \psi) \rightarrow(v t \varphi \vee v t \psi)
\end{aligned}
$$

and the following necessitation inference rule:

(NEC) necessitation for $v t$ : from $\varphi$ infer $v t \varphi$

Hájek proves that $\mathrm{BL}_{v t}$ enjoys a local deduction theorem (see Section 4.3). Moreover, he defines in the usual way a notion of $\mathrm{BL}_{v t}$-algebras, as expansions of BL-algebras with a unary operator satisfying the new axioms and rule, and proves that the logic $\mathrm{BL}_{v t}$ is complete with respect to the class of linearly ordered $\mathrm{BL}_{v t}$-algebras. Completeness also extends to any axiomatic extension of $\mathrm{BL}$, but the issue of standard completeness is left as an open problem, except for the case of Gödel logic for which it is proved.

In [38] Vychodil first introduces a logic combining both a truth-stresser and a truth-depresser. Indeed the logic $\mathrm{BL}_{v t, s t}$ is defined as an expansion of Hájek's $\mathrm{BL}_{v t}$ logic with a new unary connective "slightly true" denoted by st and with the following additional axioms

(ST1) $\varphi \rightarrow s t \varphi$,

(ST2) $s t \varphi \rightarrow \neg v t \neg \varphi$,

$(\mathrm{ST} 3) v t(\varphi \rightarrow \psi) \rightarrow(s t \varphi \rightarrow s t \psi)$ 
Note that axioms (ST2) and (ST3) put into relation both connectives $v t$ and st. Vychodil proves the completeness of the system with respect to the class of all linearly-ordered $\mathrm{BL}_{v t, s t}$-algebras (defined in the obvious way); however, he does not discuss neither its standard completeness nor the existence of some local deduction theorem. He also proposes two slightly different axiomatizations (systems I and II) for the truth-depressing hedge slightly true alone. They are defined again as expansions of BL with the unary connective st. Namely, system I has the following set of additional axioms:

$(\mathrm{ST} 1) \varphi \rightarrow s t \varphi$

$(\mathrm{ST} 4) \neg \operatorname{st}(\overline{0})$,

(ST5) $s t(\varphi \rightarrow \psi) \rightarrow(s t \varphi \rightarrow s t \psi)$,

while system II consists of the axioms (ST1), (ST4) and

$(\mathrm{ST} 6)(\varphi \rightarrow \psi) \rightarrow(s t \varphi \rightarrow s t \psi)$,

Again chain-completeness for both systems is proved, but the issues of standard completeness and (local) deduction theorem are left open.

All of these axiomatizations proposed by Hájek and Vychodil have an important common drawback: given a standard BL-chain and a hedge function (either stresser or depresser respectively) the corresponding expanded chain is not necessarily an algebraic model of the proposed logic (as it is in the fuzzy logic in broader sense). This is due to the requirement of the axiom (VT2) (in Hajék's system) or either (ST5) or (ST6) (in Vychodil systems) ${ }^{2}$ as the following examples show:

\section{Example 1.}

1. Suppose $h$ is a truth-stressing hedge function on the standard Lukasiewicz chain $[0,1]_{\mathrm{E}}$ such that there are $0<b<a<1$ such that $h(a)=a$ and $h(b)<b$. If $h(1-a+b)=1-a+b-\epsilon$ and $h(b)<b-\epsilon$, then $h(a \rightarrow b)=h(1-a+b)=1-a+b-\epsilon>1-a+h(b)=h(a) \rightarrow h(b)$, in contradiction with (VT2).

For instance, take $a=\frac{1}{2}$ and $b=\frac{1}{4}$ and the hedge function determined by the straight segments joining the following points

$$
\langle 0,0\rangle,\left\langle\frac{1}{4}, \frac{1}{10}\right\rangle,\left\langle\frac{1}{2}, \frac{1}{2}\right\rangle,\left\langle\frac{3}{4}, \frac{7}{10}\right\rangle,\langle 1,1\rangle .
$$

Then we have $h(a \rightarrow b)=h\left(\frac{3}{4}\right)=\frac{7}{10}>\frac{1}{2}+\frac{1}{10}=h(a) \rightarrow h(b)$.

Similar examples can be built for product t-norm with the necessary changes. But there are also many examples satisfying the required properties like the ones given in Section 4.3.

2. Take any truth-depressing hedge function over the standard Eukasiewicz chain $[0,1]_{\mathrm{E}}$ different from the identity, and take any point $a \in(0,1)$ such that $h(a)>a$. Then $h(a \rightarrow 0)=h(\neg a) \geq$ $\neg a>\neg h(a)=h(a) \rightarrow 0=h(a) \rightarrow h(0)$, in contradiction with both (ST5) and (ST6). ${ }^{3}$

\footnotetext{
${ }^{2}$ These axioms are analogous to the $\mathrm{K}$ axiom in modal logics (with the modal operator appearing in the place of the symbol for the truth hedge) and will be studied in Section 4.3 .

${ }^{3}$ The same result is valid for any IMTL-chain since the proof only uses the fact that the negation is involutive.
} 
3. Take the truth-depressing hedge function over the standard product chain $[0,1]_{\Pi}$ defined by straight lines with end points $\langle 0,0\rangle,\left\langle\frac{1}{2}, \frac{3}{4}\right\rangle$ and $\langle 1,1\rangle$. Then we have

$$
h\left(\frac{3}{4} \rightarrow \frac{1}{4}\right)=h\left(\frac{1}{3}\right)=\frac{1}{2}
$$

while

$$
h\left(\frac{3}{4}\right) \rightarrow h\left(\frac{1}{4}\right)=\frac{7}{8} \rightarrow \frac{3}{8}=\frac{3}{7}<\frac{1}{2},
$$

contradicting (ST5).

The examples above prove that, in general, both Hájek and Vychodil axiomatics do not cover all hedge functions. In particular, in the case of Łukasiewicz logic, while Hájek's approach still covers a quite reasonable class of truth-stressing hedge functions, the only truth-depressing hedge function admitted by Vychodil's axiomatization is the identity function. The reason seems to be that truth-stressers are similar to necessity operators in modal logic while truth-depressers are similar to possibility operators, and only necessity operators satisfy the axiom $\mathrm{K}$ in modal logic. Therefore, (VT2), despite its shortcomings, still seems more reasonable than (ST5) or (ST6).

\subsection{Some preliminaries on fuzzy logics}

In this section we gather from $[4,10,11]$ some necessary results we use in the rest of the paper. All unexplained definitions can be found in these sources.

Let $\mathrm{L}$ be a logic in a language $\mathcal{L}$. We say that L is a Rasiowa-implicative logic (c.f. [35]) if there is a binary (either primitive or definable by a formula) connective $\rightarrow$ of its language such that:

(MP) $\quad \varphi, \varphi \rightarrow \psi \vdash_{\mathrm{L}} \psi$,

(T) $\varphi \rightarrow \psi, \psi \rightarrow \chi \vdash_{\mathrm{L}} \varphi \rightarrow \chi$,

(Cong) $\varphi \rightarrow \psi, \psi \rightarrow \varphi \vdash_{\mathrm{L}} c\left(\chi_{1}, \ldots, \chi_{i}, \varphi, \ldots, \chi_{n}\right) \rightarrow c\left(\chi_{1}, \ldots, \chi_{i}, \psi, \ldots, \chi_{n}\right)$

for each $n$-ary $c \in \mathcal{L}$ and each $i<n$,

Every finitary Rasiowa-implicative logic is algebraizable in the sense of Blok and Pigozzi [5] and its equivalent algebraic semantics, the class of L-algebras, is a quasivariety; call it $\mathbb{L}$. Every L-algebra satisfies $x \rightarrow x=y \rightarrow y$ for any $x, y$, and hence the language can be expanded by a definable constant $\overline{1}=p \rightarrow p$. Then, the algebraizability gives the following strong completeness theorem:

For every set $\Gamma \cup\{\varphi\}$ of formulae, $\Gamma \vdash_{\mathrm{L}} \varphi$ iff for every $\boldsymbol{A} \in \mathbb{L}$ and every $\boldsymbol{A}$-evaluation e, $e(\varphi)=\overline{1}^{\boldsymbol{A}}$, whenever $e[\Gamma] \subseteq\left\{\overline{1}^{\boldsymbol{A}}\right\}$.

Every L-algebra $\boldsymbol{A}$ is naturally endowed with a preorder relation by setting for every $a, b \in A$ : $a \leq^{\boldsymbol{A}} b$ iff $a \rightarrow \rightarrow^{\boldsymbol{A}} b=\overline{1}^{\boldsymbol{A}}$. $\boldsymbol{A}$ is called an L-chain if $\leq^{\boldsymbol{A}}$ is a total order. ${ }^{4} \mathrm{~L}$ is called a semilinear logic iff it is strongly complete with respect to the semantics given by L-chains or, equivalently, if every L-algebra is representable as subdirect product of L-chains.

\footnotetext{
${ }^{4}$ For the sake of a lighter notation, from now on we will drop the super-indexes in the algebra operations whenever no confusion is possible.
} 
Many systems informally referred to as fuzzy logics in the literature are actually finitary Rasiowa-implicative semilinear logics. Well known examples are the three main fuzzy logics (see e.g. [20]) G (Gödel logic), $\Pi$ (Product logic) and $\mathrm{E}$ (Lukasiewicz logic), BL and SBL (the logic of all (strict) continuous t-norms, see [20,8]), WNM and NM ((Weak) Nilpotent Minimum logic, see [14]) and MTL, IMTL and SMTL (the logic of all (involutive, strict) left-continuous t-norms, see $[14,27,13]) .{ }^{5}$ A big class of fuzzy logics which are finitary, Rasiowa-implicative and semilinear, and contain the mentioned prominent examples, are the so-called core fuzzy logics: axiomatic expansions of MTL satisfying (Cong) for any possible new connective.

All core fuzzy logics enjoy a form of local deduction-detachment theorem, while some of them enjoy a globla form. We define these notions in general, because we will consider later other forms of them.

Definition 1. Let L be a Rasiowa-implicative logic. We say that $\mathrm{L}$ enjoys the local deductiondetachment theorem with respect to a set of unary terms DT if, for every set of formulae $\Gamma \cup\{\varphi, \psi\}$, it holds that $\Gamma, \varphi \vdash_{\mathrm{L}} \psi$ iff there is a term $t \in D T$ such that $\Gamma \vdash_{\mathrm{L}} t(\varphi) \rightarrow \psi$. We say that $\mathrm{L}$ enjoys the global deduction-detachment theorem if the equivalence holds in general for a fixed formula $t$.

As usual, $\varphi^{n}$ will be used as a shorthand for $\varphi \& . n . \& \varphi$, where $\varphi^{0}=\overline{1}$. Using this notation one can write the following local deduction theorem for core fuzzy logics:

Proposition 2 (Local deduction-detachment theorem for core fuzzy logics). Let L be a core fuzzy logic. For each set of formulae $\Sigma \cup\{\varphi, \psi\}$ it holds:

$$
\Sigma, \varphi \vdash_{\mathrm{L}} \psi \text { iff there is } n \in \mathrm{N} \text { such that } \Sigma \vdash_{\mathrm{L}} \varphi^{n} \rightarrow \psi \text {. }
$$

Nevertheless, some prominent fuzzy logics in enriched languages may fail to be core fuzzy logics because they may need some additional inference rules to describe the behavior of their additional connectives. Important examples are expansions with Baaz's Delta connective (see [1]). For instance, in the case of MTL, the expansion $\mathrm{MTL}_{\triangle}$ is obtained by enriching the language with the unary connective $\triangle$ and adding to the Hilbert-style system of MTL the deduction rule of necessitation (from $\varphi$ infer $\triangle \varphi$ ) and the following axiom schemata:

$$
\begin{array}{ll}
(\triangle 1) & \triangle \varphi \vee \neg \triangle \varphi \\
(\triangle 2) & \triangle(\varphi \vee \psi) \rightarrow(\triangle \varphi \vee \triangle \psi) \\
(\triangle 3) & \triangle \varphi \rightarrow \varphi \\
(\triangle 4) & \triangle \varphi \rightarrow \triangle \triangle \varphi \\
(\triangle 5) & \triangle(\varphi \rightarrow \psi) \rightarrow(\triangle \varphi \rightarrow \triangle \psi)
\end{array}
$$

The importance of these expansions has justified the introduction and usage of the class of $\triangle$ core fuzzy logics: axiomatic expansions of $\mathrm{MTL}_{\triangle}$ satisfying (Cong) for any possible new connective. Again, this is a subclass of semilinear Rasiowa-implicative logics. They satisfy the global deductiondetachment theorem in the following way:

Proposition 3 (Global deduction-detachment theorem for core fuzzy logics for $\triangle$-core fuzzy logics). For each set of formulae $\Sigma \cup\{\varphi, \psi\}$ it holds:

$$
\Sigma, \varphi \vdash_{\mathrm{MTL}_{\triangle}} \psi \text { iff } \Sigma \vdash_{\mathrm{MTL}_{\triangle}} \triangle \varphi \rightarrow \psi .
$$

\footnotetext{
${ }^{5}$ For an introduction to t-norm based fuzzy logics see e.g. [4].
} 
Now we recall a couple of definitions and results about disjunction connectives, for they provide a useful characterization of semilinearity.

A (primitive or definable) binary connective $\vee$ is called a disjunction in $\mathrm{L}$ whenever it satisfies:

(PD) $\quad \varphi \vdash_{\mathrm{L}} \varphi \vee \psi$ and $\psi \vdash_{\mathrm{L}} \varphi \vee \psi$,

$(\mathrm{PCP}) \quad$ If $\Gamma, \varphi \vdash_{\mathrm{L}} \chi$ and $\Gamma, \psi \vdash_{\mathrm{L}} \chi$, then $\Gamma, \varphi \vee \psi \vdash_{\mathrm{L}} \chi$.

Given a disjunction $\vee$ and a finitary inference rule $(R): \Gamma \vdash \varphi$ (axioms are taken as rules with $\Gamma=\emptyset)$, we define the $\vee$-form of $(R)$, denoted as $\left(R^{\vee}\right)$, as the rule $\Gamma \vee p \vdash \varphi \vee p$, where $p$ is an arbitrary propositional variable not appearing in $\Gamma \cup\{\varphi\}$.

Proposition 4. [11] Let $\mathrm{L}_{1}$ be a logic with a disjunction $\vee$ and let $\mathrm{L}_{2}$ be an expansion of $\mathrm{L}_{1}$ by a set of finitary rules $\mathcal{C}$. Then, $\vee$ is a disjunction in $\mathrm{L}_{2}$ iff $\left(R^{\vee}\right)$ holds in $\mathrm{L}_{2}$ for each $(R) \in \mathcal{C}$. In particular, $\vee$ is a disjunction in any axiomatic expansion of $\mathrm{L}_{1}$.

Proposition 5. [11] Let $\mathrm{L}$ be a finitary Rasiowa-implicative logic with a binary connective $\vee$ satisfying (PD). Consider the following two properties:

$\left(\mathrm{P}_{\vee}\right) \quad \vdash_{\mathrm{L}}(\varphi \rightarrow \psi) \vee(\psi \rightarrow \varphi)$

$\left(\mathrm{MP}_{\vee}\right) \quad \varphi \rightarrow \psi, \varphi \vee \psi \vdash_{\mathrm{L}} \psi$ and $\varphi \rightarrow \psi, \psi \vee \varphi \vdash_{\mathrm{L}} \psi$

Then the following are equivalent:

(i) $\vee$ is a disjunction and satisfies $\left(\mathrm{P}_{\vee}\right)$,

(ii) $\mathrm{L}$ is semilinear and satisfies $\left(\mathrm{MP}_{\vee}\right)$

As mentioned before, core fuzzy logics are semilinear, hence they are strongly complete with respect the class of their chains. However, this completeness may be sometimes refined to special subclasses of chains. We will use the following notions of completeness with respect to a given class of chains.

Definition $6(\mathbb{K C}, \mathrm{FS} \mathbb{K} \mathrm{C}, \mathrm{SKC})$. Let $\mathrm{L}$ be a core fuzzy logic and let $\mathbb{K}$ be a class of L-chains. We say that $\mathrm{L}$ has the (finitely) strong $\mathbb{K}$-completeness property, (F)SKKC for short, when for every (finite) set of formulae $T$ and every formula $\varphi$ it holds that $T \vdash_{\mathrm{L}} \varphi$ iff $e(\varphi)=\overline{1}^{\boldsymbol{A}}$ for each $\boldsymbol{A}$ evaluation such that $e[T] \subseteq\left\{\overline{1}^{\boldsymbol{A}}\right\}$ for every L-algebra $\boldsymbol{A} \in \mathbb{K}$. We say that $\mathrm{L}$ has the $\mathbb{K}$-completeness property, $\mathbb{K C}$ for short, when the equivalence is true for $T=\emptyset$.

Of course, the SKC implies the FSKC, and the FSKC implies the $\mathbb{K C}$. When $\mathbb{K}$ is the class of all chains whose support is the real unit interval $[0,1]$ we will denote it as $\mathcal{R}$, call its elements as real chains, and we will speak about real completeness properties. The SKC and FSKC have traditionally been proved by showing an embeddability property, namely by showing in the first case that every countable L-chain is embeddable into $\mathbb{K}$, and in the second case by showing that every countable L-chain is partially embeddable into $\mathbb{K}$ (i.e. for every finite partial of a countable L-chain there is a one-to-one mapping into some member of $\mathbb{K}$ preserving the defined operations). In [9] it was shown that these sufficient conditions are also necessary and so they provide characterizations for these completeness properties.

Theorem 1 (Characterization of completeness properties). Let L be a core fuzzy logic and $\mathbb{K} a$ class of L-chains. Then:

- L has the $\mathrm{SKC}$ iff every countable L-chain is embeddable into some member of $\mathbb{K}$.

- If the language of $\mathrm{L}$ is finite, then $\mathrm{L}$ has the $\mathrm{FSKC}$ iff every countable $\mathrm{L}$-chain is partially embeddable into $\mathbb{K}$. 


\section{Truth-stressers: a general axiomatization}

In order to solve the problems with the axiomatization of truth-stressers and depressers proposed by Hájek and Vychodil mentioned in Section 2.1, in what follows we will make use of available results decribed in Section 2.2 to obtain a simple and general axiomatizations with intuitive properties and nice completeness results. To begin with let us consider the case of truth-stressers.

Let $\mathrm{L}$ be a core fuzzy logic, and consider $\mathrm{L}_{s}$ the expansion of $\mathrm{L}$ with a new unary connective $s$ (for stresser) defined by the following additional axioms: ${ }^{6}$

(VTL1) $s \varphi \rightarrow \varphi$

(VTL2) $s \overline{1}$

and the following additional inference rule:

$\left(\mathrm{MON}_{s}\right)$ from $(\varphi \rightarrow \psi) \vee \chi$ infer $(s \varphi \rightarrow s \psi) \vee \chi$

If we denote by $\vdash_{\mathrm{L}_{s}}$ the notion of deduction defined as usual from the above axioms and rules, one can easily show the following syntactical properties.

Lemma 7. In $\mathrm{L}_{s}$ the following deductions are valid:

(i) $\vdash_{\mathrm{L}_{s}} \neg s \overline{0}$,

(ii) $\varphi \rightarrow \psi \vdash_{\mathrm{L}_{s}} s \varphi \rightarrow s \psi$,

(iii) $\psi \vdash_{\mathrm{L}_{s}} s \psi$,

(iv) $s \varphi, \varphi \rightarrow \psi \vdash_{\mathrm{L}_{s}} s \psi$.

(v) $s \varphi, s(\varphi \rightarrow \psi) \vdash_{\mathrm{L}_{s}} s \psi$.

(vi) $\varphi \vee \neg \varphi \vdash_{\mathrm{L}_{s}} \varphi \leftrightarrow s \varphi$

Proof. (i) It follows directly from (VTL1) taking $\varphi=\overline{0}$.

(ii) It follows directly from $\left(\mathrm{MON}_{s}\right)$ taking $\chi=\overline{0}$.

(iii) It follows directly from (ii) taking $\varphi=\overline{1}$ and using (VTL2).

(iv) Very easy using (ii) and modus ponens.

(v) It follows from (iv), (VTL1) and modus ponens.

(vi) One direction is indeed axiom (VTL1). For the other direction, an easy proof by cases suffices: clearly $\varphi \vdash_{\mathrm{L}_{s}} s \varphi$ and hence $\varphi \vdash_{\mathrm{L}_{s}} \varphi \rightarrow s \varphi$ as well; on the other hand, trivially $\neg \varphi \vdash_{\mathrm{L}_{s}} \varphi \rightarrow s \varphi$.

\footnotetext{
${ }^{6}$ Observe that (VTL1) coincides with Hájek's (VT1).
} 
Notice that (iv) is a kind of stronger version of the modus ponens rule: if $\varphi$ implies $\psi$, and $\varphi$ is very true, then one can derive that $\psi$ is very true as well. It must also be noted that our logics retain in the form of $(\mathrm{v})$ a deductive version of the K-like axiom used in precedent axiomatizations of logics with hedges.

On the other hand, (ii) shows that (Cong) is satisfied for the new unary connective too. Therefore, $\mathrm{L}_{s}$ is a finitary Rasiowa-implicative logic and its equivalent algebraic semantics is the class of $\mathrm{L}_{s}$-algebras.

Definition 8. An algebra $\boldsymbol{A}=\langle A, \&, \rightarrow, \wedge, \vee, \overline{0}, \overline{1}, s\rangle$ of type $\langle 2,2,2,2,0,0,1\rangle$ is an $\mathrm{L}_{s}$-algebra if it is an L-algebra expanded by a unary operator $s: A \rightarrow A$ (truth-stressing hedge) that satisfies, for all $x, y, z \in A$,

(1) $s(\overline{1})=\overline{1}$,

(2) $s(x) \leq x$,

(3) if $(x \rightarrow y) \vee z=\overline{1}$ then $(s(x) \rightarrow s(y)) \vee z=\overline{1}$.

Knowing this description of the algebraic semantics, we can prove that the logic $\mathrm{L}_{s}$ is a conservative expansion of $\mathrm{L}$ in the following strong sense.

Proposition 9 (Conservative expansion). Let $\mathcal{L}$ be the language of $\mathrm{L}$. For every set $\Gamma \cup\{\varphi\}$ of $\mathcal{L}$-formulae, $\Gamma \vdash_{\mathrm{L}_{s}} \varphi$ iff $\Gamma \vdash_{\mathrm{L}} \varphi$.

Proof. One implication is trivial. For the other one, assume that $\Gamma \nvdash_{\mathrm{L}} \varphi$. Then there exists an L-chain $\boldsymbol{A}$ and an $\boldsymbol{A}$-evaluation $e$ such that $e[\Gamma] \subseteq\{\overline{1}\}$ and $e(\varphi) \neq \overline{1}$. $\boldsymbol{A}$ can be expanded to an $\mathrm{L}_{s}$-chain $\boldsymbol{A}^{\prime}$ e.g. by defining $s(\overline{1})=\overline{1}$ and $s(a)=\overline{0}$ for every $a \in A \backslash\{\overline{1}\}$. Then $\boldsymbol{A}^{\prime}$ and $e$ provide a counterexample in the expanded language showing that $\Gamma \nvdash_{\mathrm{L}_{s}} \varphi$.

The class of $\mathrm{L}_{s}$-algebras forms a quasivariety, call it $\mathbb{L}_{s}$. Notice that if $\langle A, \&, \rightarrow, \wedge, \vee, \overline{0}, \overline{1}\rangle$ is a totally ordered L-algebra and $s: A \rightarrow A$ is any non-decreasing mapping such that $s(\overline{1})=\overline{1}$ and $s(a) \leq a$ for any $a \in A$, then the expanded structure $\langle A, \&, \rightarrow, \wedge, \vee, \overline{0}, \overline{1}, s\rangle$ is an $\mathrm{L}_{s}$-chain. ${ }^{7}$ In other words, in $\mathrm{L}_{s}$-chains the quasiequation (3) turns out to be equivalently expressed by this simplified form: if $x \rightarrow y=\overline{1}$ then $s(x) \rightarrow s(y)=\overline{1}$, and this condition simply expresses that $s$ is non-decreasing.

Moreover, since the rule $\left(\mathrm{MON}_{s}\right)$ is closed under $\vee$-forms, we know by Proposition 4 that $\vee$ remains a disjunction in the expanded logic. On the other hand, since $\left(\mathrm{P}_{\vee}\right)$ was already valid in $\mathrm{L}$, by Proposition 5 we obtain that $\mathrm{L}_{s}$ is also semilinear and hence it is complete with respect to the semantics of all $\mathrm{L}_{s}$-chains.

Theorem 2. $\mathrm{L}_{s}$ is complete with respect to the class of all $\mathrm{L}_{s}$-chains.

Corollary 10. In $\mathrm{L}_{s}$ the following deductions are valid:

$$
\begin{aligned}
& (v i) \vdash_{\mathrm{L}_{s}} s(\varphi \vee \psi) \leftrightarrow s \varphi \vee s \psi, \\
& (v i i) \vdash_{\mathrm{L}_{s}} s(\varphi \wedge \psi) \leftrightarrow s \varphi \wedge s \psi .
\end{aligned}
$$

\footnotetext{
${ }^{7}$ Observe that these three simple conditions required for $s$ would be not enough to define an $\mathrm{L}_{s}$-chain in case $\mathrm{L}_{s}$ would have been defined with the additional axiom $\mathrm{K}$ as in [21].
} 
Proof. Both properties are easily checked on $\mathrm{L}_{s}$-chains.

One might wonder whether the corresponding equation (or equations) for monotonicity of $s$, i.e. $s(x \wedge y)=s(x) \wedge s(y)$ and $s(x \vee y)=s(x) \vee s(y)$, may substitute the quasiequation (3) in the definition of $\mathrm{L}_{s}$-algebras. Notice first that over algebras satisfying (1) and (2) the equations for monotonicity are not equivalent as the following example shows. ${ }^{8}$

Example 2. Consider the finite non-linear Gödel algebra defined over $A=\{0, a, b, c, 1\}$ where 0 is the minimum, 1 is the maximum, $c$ is the only atom, $a \wedge b=c$, and $a \vee b=1$. Consider the two following truth-stresser mappings $s_{1}, s_{2}: A \rightarrow A$ (which are clearly non-decreasing, subdiagonal and preserve 1):

- Let $s_{1}(a)=s_{1}(b)=s_{1}(c)=s_{1}(0)=0$ and $s_{1}(1)=1$. So defined, $s_{1}$ satisfies the identity $s_{1}(x \wedge y)=s_{1}(x) \wedge s_{1}(y)$, but it does not satisfy the monotonicity for the supremum, indeed $s_{1}(a \vee b)=s_{1}(1)=1$ and $s_{1}(a) \vee s_{1}(b)=0 \vee 0=0$.

- Let $s_{2}(x)=x$ for every $x \neq c$ and $s_{2}(c)=0$. This mapping satisfies the monotonicity for the supremum, $s_{2}(x \vee y)=s_{2}(x) \vee s_{2}(y)$, but not for the infimum since $s_{2}(a \wedge b)=s_{2}(c)=0$ and $s_{2}(a) \wedge s_{2}(b)=a \wedge b=c$.

Now the question is whether the quasivariety $\mathbb{L}_{s}$ coincides with the variety $\mathbb{V}$ of expansions of L-algebras satisfying the equations (1), (2) and the two monotonicity equations of $s$ (observe that both classes contain the same chains). In other words, does the logic $\mathrm{L}_{s}$ coincide with the axiomatic expansion of L with the axioms $s(\varphi \vee \psi) \leftrightarrow s \varphi \vee s \psi$ and $s(\varphi \wedge \psi) \leftrightarrow s \varphi \wedge s \psi$ ? The answer is negative as shown by the following example. ${ }^{9}$

Example 3. Consider the following $\mathrm{MTL}_{s}$-algebra on the lattice over $\{0, a, b, c, d, e 1\}$ with the ordering $0<a<b<c<\{d, e\}<1\}$ specified by the following operations:

\begin{tabular}{|c|c|c|c|c|c|c|c|c|c|}
\hline$\&$ & 0 & $a$ & $b$ & $c$ & $d$ & $e$ & 1 & $x$ & $s(x)$ \\
\hline 0 & 0 & 0 & 0 & 0 & 0 & 0 & 0 & 0 & 0 \\
\hline$a$ & 0 & 0 & 0 & $a$ & $a$ & $a$ & $a$ & $a$ & 0 \\
\hline$b$ & 0 & 0 & 0 & $a$ & $a$ & $b$ & $b$ & $b$ & $b$ \\
\hline$c$ & 0 & $a$ & $a$ & $c$ & $c$ & $c$ & $c$ & $c$ & $c$ \\
\hline$d$ & 0 & $a$ & $a$ & $c$ & $d$ & $c$ & $d$ & $d$ & $d$ \\
\hline$e$ & 0 & $a$ & $b$ & $c$ & $c$ & $e$ & $e$ & $e$ & $e$ \\
\hline 1 & 0 & $a$ & $b$ & $c$ & $d$ & $e$ & 1 & 1 & 1 \\
\hline
\end{tabular}

This algebra satisfies the equations $s(x \vee y)=s(x) \vee s(y)$ and $s(x \wedge y)=s(x) \wedge s(y)$, but it does not satisfy the quasiequation corresponding to the rule $\left(M O N_{s}\right)$; indeed $(b \rightarrow a) \vee e=d \vee e=1$ while $(s(b) \rightarrow s(a)) \vee e=(b \rightarrow 0) \vee e=b \vee e=e$.

Another question is whether in the presentation of $\mathrm{L}_{s}$ the rule $\left(\mathrm{MON}_{s}\right)$ could be substituted by the following simpler rule: from $\varphi \rightarrow \psi$ infer $s \varphi \rightarrow s \psi$. This is refuted by the next example.

\footnotetext{
${ }^{8}$ We thank Franco Montagna for pointing us this observation.

${ }^{9}$ We thank Félix Bou for pointing us this example. On the other hand, this example corrects a wrong one that appeared in [15, Example 3.1.5].
} 
Example 4. If $\mathrm{L}$ is not classical propositional logic, then there must be an $\mathrm{L}$-chain $\boldsymbol{A}$ with at least three elements on its domain. Take any $a \in A \backslash\left\{\overline{0}^{\boldsymbol{A}}, \overline{1}^{\boldsymbol{A}}\right\}$, consider the direct product algebra $\boldsymbol{A} \times \boldsymbol{A}$, and expand it to an algebra $\boldsymbol{B}$ with a unary operator $s$ by putting $s(x, y)=\langle x \wedge y, x \wedge y\rangle$. An easy computation shows that $\boldsymbol{B}$ is a model of the expansion of L with (VTL1), (VTL2) and the simplified form of $\left(M O N_{s}\right)$, but $\left(M O N_{s}\right)$ itself is not sound. Indeed, we have $(\langle\overline{1}, \overline{1}\rangle \rightarrow\langle\overline{1}, a\rangle) \vee\langle a, \overline{1}\rangle=$ $\langle\overline{1}, a\rangle \vee\langle a, \overline{1}\rangle=\langle\overline{1}, \overline{1}\rangle$, while $(s(\overline{1}, \overline{1}) \rightarrow s(\overline{1}, a)) \vee\langle a, \overline{1}\rangle=(\langle\overline{1}, \overline{1}\rangle \rightarrow\langle a, a\rangle) \vee\langle a, \overline{1}\rangle=\langle a, a\rangle \vee\langle a, \overline{1}\rangle=$ $\langle a, \overline{1}\rangle \neq\langle\overline{1}, \overline{1}\rangle$.

Similarly, inspired by the well-known presentation of logics with $\triangle$, one might also ask whether $\left(\mathrm{MON}_{s}\right)$ could be substituted by the globalization rule for $s$ : from $\varphi$ infer $s \varphi$. The answer is again negative as it is obvious using the last example.

Therefore, we have obtained an axiomatization for fuzzy logics $\mathrm{L}_{s}$ with stressing hedges which, as shown by the previous examples, cannot be readily simplified. The next natural issue to consider is whether the completeness of $\mathrm{L}_{s}$ can be restricted to some distinguished semantics of $\mathrm{L}_{s}$-chains. Capitalizing on the characterization of completeness properties (Theorem 1), we will see that $\mathrm{L}_{s}$ has exactly the same good completeness properties as its underlying logic L.

Theorem 3 (Finite strong real completeness). Let L be a core fuzzy logic in a finite language. L has the $\mathrm{FSRC}$ if, and only if, $\mathrm{L}_{s}$ has the $\mathrm{FSRC} .{ }^{10}$

Proof. The implication from right to left follows directly from the fact that $\mathrm{L}_{s}$ is a conservative expansion of $\mathrm{L}$ (Proposition 9). Assume now that $\mathrm{L}$ has the FSRC. Take any $\mathrm{L}_{s}$-chain $\boldsymbol{A}=$ $\langle A, \&, \rightarrow, \wedge, \vee, \overline{0}, \overline{1}, s\rangle$ and let $B$ be a finite partial subalgebra of $\boldsymbol{A}$. We have to show that there exists a standard $\mathrm{L}_{s}$-chain $\left\langle[0,1], \wedge, \vee, *, \Rightarrow, s^{\prime}, 0,1\right\rangle$ and a mapping $f: B \rightarrow[0,1]$ preserving the existing operations. By Theorem 1, using the necessity of the embeddability property, we know that the $s$-free reduct of $\boldsymbol{A}$ is partially embeddable into a standard L-chain $\langle[0,1], \wedge, \vee, *, \Rightarrow, 0,1\rangle$. Denote this embedding by $f$ and consider any non-decreasing and subdiagonal function $s^{\prime}:[0,1] \rightarrow$ $[0,1]$ satisfying $s^{\prime}(f(x))=f(s(x))$ for every $x \in B$ such that $s(x) \in B$. There are obviously many such functions $s^{\prime}$ interpolating the set of points $P=\{\langle f(x), f(s(x))\rangle \mid x, s(x) \in B\}$, for instance a piecewise linear interpolant. Another interpolant can be defined as follows: let $0=z_{1}<\ldots<$ $z_{n}<1$ be the set of elements of $[0,1]$ such that $\left\langle z_{i}, x\right\rangle \in P$ for some $x$ and define $s^{\prime}(1)=1$ and, for all $z \in[0,1)$,

$$
s^{\prime}(z)=f\left(s\left(x_{i}\right)\right) \text {, if } z_{i} \leq z<z_{i+1}
$$

where $x_{i} \in B$ is such that $z_{i}=f\left(x_{i}\right)$. In any case $s^{\prime}$ makes $\left\langle[0,1], \wedge, \vee, *, \Rightarrow, s^{\prime}, 0,1\right\rangle$ an $\mathrm{L}_{s^{-}}$-chain and $f$ a partial embedding of $\mathrm{L}_{s}$-chains.

Actually, this theorem can be generalized to arbitrary classes of L-chains and their $s$-expansions, proved in a completely analogous way, and yielding a more general result.

Corollary 11. Let $\mathrm{L}$ be a core fuzzy logic in a finite language, $\mathbb{K}$ a class of $\mathrm{L}$-chains, and $\mathbb{K}_{s}$ the class of the $\mathrm{L}_{s}$-chains whose s-free reducts are in $\mathbb{K}$. Then $\mathrm{L}$ has the $\mathrm{FSKC}$ if, and only if, $\mathrm{L}_{s}$ has the $\mathrm{FSK}_{s} \mathrm{C}$.

\footnotetext{
${ }^{10}$ The assumption of a finite language is necessary to assure that the FSRC property is equivalent to the partial embedding property result used in the proof. In fact, we do not know whether the theorem would hold without this assumption.
} 
Observe that the implication from right to left in the last theorem and corollary does not need the assumption of finiteness of the language. For the strong real completeness we obtain an analogous result (which again does not need that assumption).

Theorem 4 (Strong real completeness). Let L be a core fuzzy logic. Then $\mathrm{L}$ has the SRC if, and only if, $\mathrm{L}_{s}$ has the $\mathrm{SRC}$.

Proof. Again one implication just follows from the fact that $\mathrm{L}_{s}$ is a conservative expansion of $\mathrm{L}$. For the converse one assume that $\mathrm{L}$ has the $\mathrm{SRC}$. We have to show that any countable $\mathrm{L}_{s}$-chain can be embedded into a standard $\mathrm{L}_{s}$-chain. Let $\boldsymbol{A}$ be a countable $\mathrm{L}_{s}$-chain. By Theorem 1, we know that the $s$-free reduct of $\boldsymbol{A}$ is embeddable into a standard L-chain $\boldsymbol{B}=\langle[0,1], *, \Rightarrow, \wedge, \vee, 0,1\rangle$. Denote this embedding by $f$ and define $s^{\prime}: B \rightarrow B$ in the following way: for each $z \in[0,1]$, $s^{\prime}(z)=\sup \{f(s(x)) \mid x \in A, f(x) \leq z\}$. So defined, $s^{\prime}$ is a non-decreasing and subdiagonal function such that $s^{\prime}(f(x))=f(s(x))$ for any $x \in A$ and hence $\boldsymbol{B}$ expanded with $s^{\prime}$ is a standard $\mathrm{L}_{s}$-chain where $\boldsymbol{A}$ is embedded.

Observe that the proof of the previous theorem can be repeated whenever the linear order of the chains is complete. Therefore we obtain the following corollary.

Corollary 12. Let $\mathrm{L}$ be a core fuzzy logic, $\mathbb{K}$ a class of completely ordered L-chains, and $\mathbb{K}_{s}$ the class of the $\mathrm{L}_{s}$-chains whose s-reducts are in $\mathbb{K}$. Then $\mathrm{L}$ has the $\mathrm{SKC}$ if, and only if, $\mathrm{L}_{s}$ has the $\mathrm{SK}_{s} \mathrm{C}$.

\section{Deduction theorems and the variety problem}

In Section 2.2 we have seen that if $\mathrm{L}$ is a core fuzzy logic, then $\mathrm{L}_{s}$ is a finitary Rasiowa-implicative logic whose equivalent algebraic semantics is the quasivariety of $\mathrm{L}_{s}$-algebras, $\mathbb{L}_{s}$. Moreover, $\mathrm{L}_{s}$ is a semilinear logic (complete with respect to chains of $\mathbb{L}_{s}$ ). However, we do not know whether $\mathbb{L}_{s}$ always forms a variety. In this section we present some families of logics $\mathrm{L}_{s}$ enjoying a local (global) form of deduction-detachment theorem. As a consequence, by virtue of the next theorem, we obtain that the quasivarieties associated to these families of logics are, in fact, varieties.

Theorem 5. Let $\mathrm{L}$ be a core fuzzy logic. Assume that $\mathrm{L}_{s}$ enjoys the local deduction-detachment theorem with respect to a set DT. Then the class of $\mathrm{L}_{s}$-algebras is a variety.

Proof. First observe that the local deduction-detachment theorem entails that for every $t \in D T$, $\vdash_{\mathrm{L}_{s}} t(\overline{1})$. Indeed, if $p$ is a propositional variable and $t \in D T$, we have $\vdash_{\mathrm{L}_{s}} t(p) \rightarrow t(p)$, and by the local deduction-detachment theorem, $p \vdash_{\mathrm{L}_{s}} t(p)$. By structurality, we have $\overline{1} \vdash_{\mathrm{L}_{s}} t(\overline{1})$ and hence $\vdash_{\mathrm{L}_{s}} t(\overline{1})$. Let $\boldsymbol{A}$ be another algebra obtain by adding a new element $c$ to $\boldsymbol{B}_{4}$ such that $a \vee b=c<1$.

Now let $\mathbb{K}$ be the variety axiomatized by the following equations:

(a) an equational base of $\mathbb{L}$,

(b) $s(\overline{1})=\overline{1}$,

(c) $s(x) \wedge x=s(x)$,

(d) $t((\varphi \rightarrow \psi) \vee \chi) \rightarrow((s(\varphi) \rightarrow s(\psi)) \vee \chi)=\overline{1}$, for each $\varphi, \psi$, $\chi$ where $t \in D T$ is their corresponding deduction term. 
We check that the class of $\mathrm{L}_{s}$-algebras coincides with $\mathbb{K}$. If $\boldsymbol{A} \in \mathbb{L}_{s}$, it is clear that it satisfies all the equations because they correspond to theorems of $\mathrm{L}_{s}$. Conversely, take an algebra $\boldsymbol{A} \in \mathbb{K}$. By (a), we know that its $s$-free reduct is an L-algebra. Since it also satisfies (b) and (c), we only have to check the validity of the quasiequation: if $(x \rightarrow y) \vee z=\overline{1}$ then $(s(x) \rightarrow s(y)) \vee z=\overline{1}$. Assume that $e$ is an $\boldsymbol{A}$-evaluation and $(e(x) \rightarrow e(y)) \vee e(z)=\overline{1}$. We know that $(x \rightarrow y) \vee z \vdash_{\mathrm{L}_{s}}(s(x) \rightarrow s(y)) \vee z$. By the local deduction-detachment theorem, there is $t \in D T$ such that $\vdash_{\mathrm{L}_{s}} t((x \rightarrow y) \vee z) \rightarrow$ $(s(x) \rightarrow s(y)) \vee z$, and hence $\boldsymbol{A}$ satisfies $t((x \rightarrow y) \vee z) \rightarrow(s(x) \rightarrow s(y)) \vee z=\overline{1}$. From this, we obtain $t(\overline{1}) \rightarrow(s(e(x)) \rightarrow s(e(y))) \vee e(z)=\overline{1}$, and hence $(s(e(x)) \rightarrow s(e(y))) \vee e(z)=\overline{1}$.

\subsection{Logics of a finite BL-chain and related cases}

The first family we consider is that of the logics $\mathrm{L}_{s}$ where $\mathrm{L}$ is the logic of a finite BL-chain $\boldsymbol{C}$ having $n$ elements, i.e. $\boldsymbol{C}$ is an ordinal sum of copies of finite MV-chains $\left(\mathbf{E}_{k}\right)$ and finite Gödel-chains $\left(\boldsymbol{G}_{r}\right)$.

Lemma 13. Assume that a core fuzzy logic $\mathrm{L}$ is the logic of a finite BL-chain $\boldsymbol{C}$, i.e. the class of $\mathrm{L}-$ algebras is the variety generated by $\boldsymbol{C}$. Then:

- L-chains are exactly the subalgebras of $\boldsymbol{C}$.

- Given a BL-filter $F$ of $\boldsymbol{C}$ its corresponding congruence defined $\equiv_{F}$ can be described as: $x \equiv_{F} y$ iff either $x=y$ or $x, y \in F$, i.e. the congruence classes are $F$ and the singletons $\{x\}$ for any $x \notin F$.

- The set of $\mathrm{L}_{s}$-filters of $\boldsymbol{C}$ coincides with the set of BL-filters that are closed under $s$.

Proof. The first claim is a consequence of [12, Theorem 1], taking into account that every finite BL-chain is subdirectly irreducible and the fact that any chain belonging to the variety generated by a finite Gödel or MV-chain is a subalgebra of it.

The proof of the second statement is easy. Recall that if $x \geq y$, then $x \equiv_{F} y$ iff $x \rightarrow y \in F$. On the other hand, the filters of $\boldsymbol{C}$ are the principal filters generated by an element $a$ that either belongs to a Gödel component or is the bottom of an MV component. Thus, an easy computation shows that $x \rightarrow y \in F$ iff either $x=y$ or $x, y \in F$.

In order to prove the third statement observe first that if $F$ is an $\mathrm{L}_{s}$-filter of $C$, then it is closed under $s$ since if $a \in F$, then $\overline{1} \rightarrow a \in F$ and thus $\overline{1} \rightarrow s(a)=s(a) \in F$. For the converse, suppose that $F$ is a BL-filter closed under $s$ and assume that $a \rightarrow b \in F$. If $a \leq b$, by monotonicity we have $s(a) \leq s(b)$ and hence $s(a) \rightarrow s(b)=\overline{1} \in F$. If $a>b$, we have $a \equiv_{F} b$ and thus, by the previous statement $a, b \in F$. Since $F$ is closed under $s, s(a), s(b) \in F$ and, again by the previous statement $s(a) \equiv_{F} s(b)$, which gives $s(a) \rightarrow s(b) \in F$.

Lemma 14. Let $\mathrm{L}$ be the core fuzzy logic of a finite $\mathrm{BL}-$ chain $\boldsymbol{C}$. Then, in any $\mathrm{L}_{s}$-algebra $\boldsymbol{A}$, for every principal $\mathrm{L}_{s}$-filter $F i(\bar{a})$ generated by an element $\bar{a} \in \boldsymbol{A}$ there is an element $t(\bar{a})$ such that $F i(\bar{a})=\left[t(\bar{a}), \overline{1}^{\boldsymbol{A}}\right]$.

Proof. Assume that $\boldsymbol{C}$ has $n$ elements, $k$ components in its ordinal sum decomposition, and $m$ is the maximum length of its MV components. If $\boldsymbol{A}$ is an $\mathrm{L}_{s}$-algebra, then $\boldsymbol{A}$ can be embedded into a direct product $\prod_{i \in I} \boldsymbol{C}$ (remember that any $\mathrm{L}_{s}$-chain is a subalgebra of $\boldsymbol{C}$ ). Given an element $\bar{a} \in A$ take the element $t(\bar{a})=\left(s^{n}\left(. k . s^{n}\left(\bar{a}^{m}\right)\right)^{m} \ldots\right)^{m}$. An easy computation shows that $t(\bar{a})$ is idempotent and fixed by $s$. Then we can prove that $F i(\bar{a})$ coincides with the principal lattice filter 
generated by $t(\bar{a})$. The proof follows from the following facts: (1) $t(\bar{a}) \in F i(\bar{a}),(2)$ if $t(\bar{a})_{i}$ is the $i$-projection of $t(\bar{a})$, then $F i\left(t(\bar{a})_{i}\right)=\left\{x \in C \mid x \geq t(\bar{a})_{i}\right\}$ is the filter of $C$ generated by $t(\bar{a})_{i}$, and (3) by definition $F i(t(\bar{a}))=\prod_{i \in I} F i\left(t(\bar{a})_{i}\right) \cap A$.

Theorem 6. Let $\mathrm{L}$ be the core fuzzy logic of a finite BL-chain $\boldsymbol{C}$. Assume that $\boldsymbol{C}$ has $n$ elements, $k$ components in its ordinal sum decompostion, and $m$ is the maximum length of its MV components. Consider the term $t(p)=\left(s^{n}\left(. k . s^{n}\left(p^{m}\right)\right)^{m} \ldots\right)^{m}$. Then the logic $\mathrm{L}_{s}$ enjoys the glocal deductiondetachment theorem w.r.t. $t(p)$, i.e. for every set $\Gamma \cup\{\varphi, \psi\}$ of formulae

$$
\Gamma, \varphi \vdash_{\mathrm{L}_{s}} \psi \text { iff } \Gamma \vdash_{\mathrm{L}_{s}} t(\varphi) \rightarrow \psi .
$$

Proof. The right-to-left direction follows easily from the observation that $\varphi \vdash_{\mathrm{L}_{s}} t(\varphi)$. Let us prove the converse direction by a semantical reasoning using completeness, i.e. we assume $\Gamma, \varphi \models_{\mathbb{L}_{s}} \psi$ and we have to show $\Gamma \models_{\mathbb{L}_{s}} t(\varphi) \rightarrow \psi$. Take any $\mathrm{L}_{s}$-chain $\boldsymbol{A}$ and any $\boldsymbol{A}$-evaluation $e$ such that $e[\Gamma] \subseteq$ $\left\{\overline{1}^{\boldsymbol{A}}\right\}$. Consider the matrix $\mathrm{L}_{s}$-model $\langle\boldsymbol{A}, F i(e[\Gamma], e(\varphi))\rangle$. By soundness $e(\psi) \in F i(e[\Gamma], e(\varphi))$, i.e. $e(\psi) \in F i(e(\varphi))=\left[t(e(\varphi)), \overline{1}^{\boldsymbol{A}}\right]$. Therefore, $t(e(\varphi)) \leq e(\psi)$ and so $e(t(\varphi) \rightarrow \psi)=\overline{1}^{\boldsymbol{A}}$.

Now, using Theorem 5, we immediately obtain the next corollary.

Corollary 15. If $\mathrm{L}$ is the logic of a finite $\mathrm{BL}$-chain, the quasivariety associated to the logic $\mathrm{L}_{s}$ is a variety.

Some remarks are in order here:

- Lemma 14 and Theorem 6 can be easily generalized to any logic L induced by a finite MTLchain provided that the $\mathrm{L}_{s}$-filters on $\mathrm{L}_{s}$-chains coincide with the MTL-filters closed under $s$.

- A sufficient condition for an MTL-filter on an $\mathrm{L}_{s}$-chain closed under $s$ to be an $\mathrm{L}_{s}$-filter is the fact that $a \equiv_{F} b$ iff either $a=b$ or $a, b \in F$. For example, any finite WNM-chain $\boldsymbol{C}$ (with $n$ elements) satisfies this condition, and hence the logic $\mathrm{L}_{s}$ enjoys the global deduction-detachment theorem (w.r.t. the term $\left.t(\varphi)=\left(s^{n}(\varphi)\right)^{2}\right)$ and thus the quasivariety corresponding to the logic of a finite WNM-chain with a truth-stresser is a variety.

- The following example proves that there are finite MTL-chains with MTL-filters closed under $s$ that are not $\mathrm{L}_{s}$-filters.

Example 5. Take a finite chain $\boldsymbol{A}$ of 6 elements $(1>a>b>c>d>0)$ and define the operation $*$ by (assuming that $*$ is determined when one value is 0 or 1) $a * a=a$, and $x * y=d$ otherwise. Then the MTL-filters are $\{1\},\{1, a\},\{1, a, b, c, d\}$ and $A$ itself. Define the operator $s$ by (the values of 0 and 1 are determined) $s(a)=a, s(b)=b, s(c)=s(d)=0$. It is obvious that the MTL-filters closed under $s$ are $\{1\},\{1, a\}$ and $A$. But $\{1, a\}$ is not an $\mathrm{L}_{s}$-filter since $b \rightarrow c=a$ and $s(b) \rightarrow s(c)=b \rightarrow 0=0 \notin\{1, a\}$.

\subsection{Logics with $\triangle$}

We consider now the case of logics $\mathrm{L}_{s}$ based on a $\triangle$-core fuzzy logic L, i.e. where the projection $\triangle$ operator is definable. In this case the situation is much simpler because, in fact, the resulting logic is an axiomatic expansion of $\mathrm{L}$ : 
Proposition 16. Let $\mathrm{L}$ be $\triangle$-core fuzzy logic. Then $\mathrm{L}_{s}$ is the axiomatic expansion of $\mathrm{L}$ obtained by adding:

$$
\begin{aligned}
& \text { (VTL1) } s \varphi \rightarrow \varphi, \\
& (\text { VTL2) } s \overline{1}, \\
& \left(M O N_{\triangle}\right) \triangle(\varphi \rightarrow \psi) \rightarrow(s \varphi \rightarrow s \psi) .
\end{aligned}
$$

Proof. Let $\mathrm{L}^{\prime}$ be the axiomatic expansion of $\mathrm{L}$ given by these three axioms. It is clear that, thanks to $\left(\mathrm{MON}_{\triangle}\right)$, the new connective satisfies the congruence condition (Cong) and hence, since $\mathrm{L}^{\prime}$ is an axiomatic expansion of a semilinear logic, it remains semilinear. Then, using that both $\mathrm{L}^{\prime}$ and $\mathrm{L}_{s}$ are complete with respect to their corresponding chains, it is easy to check that each one validates the axioms and rules of the other and thus they are the same logic.

Therefore, in this case $\mathrm{L}_{s}$ is a $\triangle$-core fuzzy logic itself and hence it satisfies the global deductiondetachment theorem in the form of Proposition 3 and, by Theorem 5 the class of $\mathrm{L}_{s}$-algebras is a variety.

$\triangle$-core fuzzy logics include the $n$-valued Lukasiewicz logic $\mathrm{E}_{n}$ or the axiomatic extensions of MTL by the axiom $\neg(\varphi)^{n} \vee \varphi$, called $\mathrm{S}_{n}$ MTL (see [26]). In both cases $\triangle \varphi$ is defined as $\varphi^{n}$ and they define a sequence of nested logics starting with classical propositional calculus: CPC $=\mathrm{\iota}_{2} \subseteq \mathrm{\iota}_{3} \subseteq$ $\ldots \subseteq \mathrm{L}_{n} \subseteq \ldots$ and $\mathrm{CPC}=\mathrm{S}_{2} \mathrm{MTL} \subseteq \mathrm{S}_{3} \mathrm{MTL} \subseteq \ldots \subseteq \mathrm{S}_{n} \mathrm{MTL} \subseteq \ldots$ respectively. On the other hand, given a core fuzzy logic L, one can also consider the family of axiomatic extensions of $\mathrm{L}_{s}$ with the axiom $\neg\left(s^{n}\left(. \stackrel{n}{.} .\left(s^{n}\left(\varphi^{n}\right)\right)^{n} \ldots\right)\right)^{n} \vee \varphi$, where $\triangle$ is definable as $\left(s^{n}\left(. n .\left(s^{n}\left(\varphi^{n}\right)\right)^{n} \ldots\right)\right)^{n}$. With these logics, denoted $\mathrm{S}_{n} \mathrm{~L}_{s}$, we obtain again a sequence of nested logics $\mathrm{S}_{2} \mathrm{~L}_{s} \subseteq \mathrm{S}_{3} \mathrm{~L}_{s} \subseteq \ldots \subseteq \mathrm{S}_{n} \mathrm{~L}_{s} \subset \ldots$

\subsection{Logics satisfying the modal axiom $K$ for the truth-stresser}

The third family we consider is the family of logics $\mathrm{L}_{s K}$ defined as the axiomatic extensions of the $\operatorname{logics} \mathrm{L}_{s}$ with the following axiom:

$(\mathrm{VT} 2) s(\varphi \rightarrow \psi) \rightarrow(s \varphi \rightarrow s \psi)$

Axiom (VT2) is the well-known axiom $K$ of modal logics for the truth-stresser connective $s$, and it is one of the axioms of Hájek's $\mathrm{BL}_{v t}$ logic [21]. In our setting it means that the logic $\mathrm{L}_{s K}$ requires that if both $\varphi$ and $\varphi \rightarrow \psi$ are "very true" then so is $\psi$.

It turns out that the presence of axiom (VT2) in $\mathrm{L}_{s K}$ allows for an equivalent axiomatic presentation where the rule of inference $\left(\mathrm{MON}_{s}\right)$ can be replaced by the weaker rule of necessitation for $s$ :

$\left(\mathrm{NEC}_{s}\right)$ from $\varphi$ infer $s \varphi$

Lemma 17. Let $\mathrm{L}_{s K}^{\prime}$ be the logic obtained from $\mathrm{L}_{s K}$ by adding the axiom

$$
\text { (VT3) } s(\varphi \vee \psi) \rightarrow(s \varphi \vee s \psi) \text {. }
$$

and replacing the rule $\left(M O N_{s}\right)$ by the rule $\left(N E C_{s}\right)$. Then the rule $\left(M O N_{s}\right)$ is derivable in $\mathrm{L}_{s K}^{\prime}$.

Proof. From $(\varphi \rightarrow \psi) \vee \chi$, using necessitation and axiom (VT3) we infer $s(\varphi \rightarrow \psi) \vee s \chi$, by (VTL1) we then infer $s(\varphi \rightarrow \psi) \vee \chi$, and by (VT2) we finally infer $(s \varphi \rightarrow s \psi) \vee \chi$. 
Notice that, by a simple inspection, the logic $\mathrm{L}_{s K}^{\prime}$ indeed corresponds to Hájek's $\mathrm{BL}_{v t}$ logic when $\mathrm{L}=\mathrm{BL}$. Moreover, since both the axiom (VT3) and the necessitation rule $\left(\mathrm{NEC}_{s}\right)$ are already derivable in $\mathrm{L}_{s K}$, it follows from the above lemma that the logics $\mathrm{L}_{s K}^{\prime}$ and $\mathrm{L}_{s K}$ are indeed equivalent.

Now, following Hájek in [21], we prove a local deduction-detachment theorem for $\mathrm{L}_{s K}$ (similar to the one proved for $\triangle$ ). We will need an auxiliary notation: $\tau \varphi$ stands for $s(\varphi \& \varphi)$ and $\tau^{n} \varphi$ stands for $\tau(. \stackrel{n}{.} \tau(\tau \varphi) . \stackrel{n}{n})$.

Lemma 18 (cf. [21])). In $\mathrm{L}_{s K}$ the following formulae are provable:

(i) $\tau^{n+1} \varphi \rightarrow \tau^{n} \varphi$

(ii) $\tau \varphi \rightarrow s \varphi, \tau \varphi \rightarrow \varphi \& \varphi$

(iii) $\tau(\varphi \vee \psi) \leftrightarrow(\tau \varphi \vee \tau \psi)$.

Theorem 7 (Local deduction-detachment theorem for $\mathrm{L}_{s K}$ ). Let $T$ be a theory and let $\varphi, \psi$ be formulae. Then: $T \cup\{\varphi\} \vdash_{\mathrm{L}_{s K}} \psi$ iff $T \vdash_{\mathrm{L}_{s K}} \tau^{n} \varphi \rightarrow \psi$ for some $n$.

Proof. The proof is by induction as usual. Let us check the induction step for deduction rules. If $T \vdash_{\mathrm{L}_{s K}} \tau^{n} \varphi \rightarrow \alpha$ and $T \vdash_{\mathrm{L}_{s K}} \tau^{n} \varphi \rightarrow(\alpha \rightarrow \beta)$, then $T \vdash_{\mathrm{L}_{s K}}\left(\tau^{n} \varphi \& \tau^{n} \varphi\right) \rightarrow \beta$, thus $T \vdash_{\mathrm{L}_{s K}}$ $\tau^{n+1} \varphi \rightarrow \beta$. Similarly, if $T \vdash_{\mathrm{L}_{s K}} \tau^{n} \varphi \rightarrow \beta$, then $T \vdash_{\mathrm{L}_{s K}} s\left(\tau^{n} \varphi\right) \rightarrow s \beta$, thus $T \vdash_{\mathrm{L}_{s K}} \tau^{n+1} \varphi \rightarrow$ $s \beta$.

The corresponding algebraic structures are the $\mathrm{L}_{s K^{-}}$-algebras. An algebra $\boldsymbol{A}=\langle A, \&, \rightarrow$ $, \wedge, \vee, \overline{0}, \overline{1}, s\rangle$ is an $\mathrm{L}_{s K}$-algebra if it is an L-algebra expanded by a unary operator $s$ (stressing hedge) that satisfies, for all $x, y \in A$,

(ve1) $s(x) \leq x$,

$($ ve2) $s(x \rightarrow y) \leq(s(x) \rightarrow s(y))$,

$($ ve3) $s(x \vee y) \leq(s(x) \vee s(y))$,

(ve4) $s(\overline{1})=\overline{1}$.

From the above remarks, an $\mathrm{L}_{s K^{-}}$-algebra is just an $\mathrm{L}_{s}$-algebra further satisfying the property (ve2). Therefore, in this case it is obvious that $\mathrm{L}_{s K}$-algebras form a variety (recall that, as in $\triangle$-core fuzzy logics, the inference rules of the logic are modus ponens and necessitation). On the other hand, as usual, for each left-continuous t-norm $*$, the chain obtained by adding to $[0,1]_{*}$ a truth-stressing hedge $s$ satisfying the above properties is a real $\mathrm{L}_{s K}$-chain.

Next we give some examples of truth-stressers on real chains $[0,1]_{*}$ satisfying axiom (VT2), we will call them $K$-truth-stressers.

Example 6. 1. The function $s(x)=x * . . . * x$ ( $x^{n}$ for short) is a K-truth-stressing function over $[0,1]_{*}$ for any left-continuous $t$-norm $*$. Obviously this truth-stressing function is continuous if the $t$-norm is so and it is the identity if the t-norm is the minimum.

2. The function $s(x)=x \cdot x$ (product of reals) is also a $K$-truth-stressing function for the three basic continuous t-norms. Observe that this function coincides with the one of the previous example for $*$ being the product $t$-norm and $n=2$. 
3. The function defined by Eukasiewicz t-norm as $s(x)=x *_{\mathrm{E}} x=\max \{0,2 x-1\}$ is a K-truthstressing function for Eukasiewicz and minimum t-norms but not for product. This function coincides with the first example for Eukasiewicz $t$-norm and $n=2$.

4. For any $k \in[0,1]$, the function $s(x)=k \cdot x$ for $x<1$ and $s(1)=1$ is a $K$-truth-stressing function for the three basic continuous t-norms. Observe that when $k=0$, this is the $\triangle$ operator.

Since it is an axiomatic extension of $\mathrm{L}_{s}$, the logic $\mathrm{L}_{s K}$ is semilinear and so it is complete with respect to the variety of $\mathrm{L}_{s K^{-}}$-algebras and with respect to the class of $\mathrm{L}_{s K}$-chains.

The problem of standard completeness for the logics $\mathrm{L}_{s K}$ is far from being solved. In the case $\mathrm{L}$ is the logic of a Gödel chain (for continuous t-norms) or, more in general, a WNM-chain (for the general MTL-chains) the problem is easy since we have the following result. ${ }^{11}$

Proposition 19. Let $\mathrm{L}$ be the logic of a WNM-chain. ${ }^{12}$ Then the logic $\mathrm{L}_{s K}$ coincides with the $\operatorname{logic} \mathrm{L}_{s}$.

Proof. It is only necessary to prove that axiom (VT2) is valid over each $\mathrm{L}_{s}$-chain and this is easy because if $a \leq b$ then $s(a \rightarrow b)=1=s(a) \rightarrow s(b)$, and if $a>b$ then either $s(a)=s(b)$, and then $s(a \rightarrow b)=s(a) \rightarrow s(b)=1$, or $s(a \rightarrow b)=s(\neg a \vee b)=s(\neg a) \vee s(b) \leq \neg s(a) \vee s(b)=s(a) \rightarrow s(b)$ (take into account that $s(\neg a) \leq \neg a \leq \neg s(a)$ ).

Then, by theorems 3 and 4, we obtain the following result:

Corollary 20. Let $\mathrm{L}$ be the logic of a WNM-chain. Then the $\mathrm{L}_{s K}$ is (finite) strong real complete whenever $\mathrm{L}$ is (finite) strong real complete.

If $\mathrm{L}$ is the logic of a continuous t-norm, the only one such that $\mathrm{L}_{s}$ satisfies (VT2) is Gödel logic, and thus it is strong real complete. For the rest of logics of continuous t-norms, including $\mathrm{E}_{s K}$ and $\Pi_{s K}$, the problem of their real completeness remains open.

\section{The case of truth-depressers}

Very similarly to the case of truth-stressers, we can proceed to define an axiomatization for the case of truth-depressers just by replacing axioms (VTL1) and (VTL2) with dual versions (STL1) and (STL2) (ST for slightly true). Namely, given a core fuzzy logic L, we define $\mathrm{L}_{d}$ as the expansion of $\mathrm{L}$ with a new unary connective $d$, the following additional axioms ${ }^{13}$

$$
\begin{aligned}
& (\mathrm{STL} 1) \varphi \rightarrow d \varphi, \\
& (\mathrm{STL} 2) \neg d \overline{0},
\end{aligned}
$$

and the following additional inference rule

$$
\left(\mathrm{MON}_{d}\right) \text { from }(\varphi \rightarrow \psi) \vee \chi \operatorname{infer}(d \varphi \rightarrow d \psi) \vee \chi
$$

\footnotetext{
${ }^{11}$ Already given by Hájek [21] for the case of L being Gödel logic.

${ }^{12}$ Recall that a Gödel chain is a particular case of WNM-chain. Recall as well that in a WNM-chain, the operations are defined as follows: $a \& b=\min \{a, b\}$ if $a>\neg b$ and $a * b=0$ otherwise; $a \rightarrow b=1$ if $a \leq b$ and $a \rightarrow b=\neg a \vee b$ otherwise.

${ }^{13}$ Observe that (STL1) and (ST2) coincide with Vychodil's (ST1) and (ST4) respectively.
} 
Being a kind of dual version of $\mathrm{L}_{s}$, many properties are proved in a completely analogous way:

Lemma 21. In $\mathrm{L}_{d}$ the following deductions are valid:
(i) $\vdash_{\mathrm{L}_{d}} d \overline{1}$
(ii) $\varphi \rightarrow \psi \vdash_{\mathrm{L}_{d}} d \varphi \rightarrow d \psi$,
(iii) $\neg \varphi \vdash_{\mathrm{L}_{d}} \neg d \varphi$,
(iv) $\vdash_{\mathrm{L}_{d}} \neg d \varphi \rightarrow \neg \varphi$,
(v) $d \varphi, \varphi \rightarrow \psi \vdash_{\mathrm{L}_{d}} d \psi$.
(vi) $\varphi \vee \neg \varphi \vdash_{\mathrm{L}_{s}} \varphi \leftrightarrow d \varphi$

Proof. (i) It follows directly from (STL1) taking $\varphi=\overline{0}$.

(ii) It follows directly from $\left(\mathrm{MON}_{d}\right)$ taking $\chi=\overline{0}$.

(iii) It follows from (ii) for $\psi=\overline{0}$ and (STL2).

(iv) It follows directly from (STL1) using the fact that $(\varphi \rightarrow \psi) \rightarrow(\neg \psi \rightarrow \neg \varphi)$ is derivable in MTL.

(v) Very easy using (ii) and modus ponens.

(vi) Analogous to (vi) of Lemma 7 with the obvious modifications.

It is interesting to remark that (v) provides a kind of weaker or modified version of modus ponens with the truth-depresser: if $\varphi$ implies $\psi$, and $\varphi$ is slightly true, then one can derive that $\psi$ is slightly true as well.

Again, (ii) shows that the (Cong) condition is satisfied by the new unary connective too. Therefore, the logic $\mathrm{L}_{d}$ is Rasiowa-implicative and its equivalent algebraic semantics is the class of $\mathrm{L}_{d}$-algebras. An algebra $\boldsymbol{A}=\langle A, \&, \rightarrow, \wedge, \vee, \overline{0}, \overline{1}, s\rangle$ of type $\langle 2,2,2,2,1,0,0\rangle$ is an $\mathrm{L}_{d}$-algebra if it is an L-algebra expanded by a unary operator $d: A \rightarrow A$ (truth-depressing hedge) that satisfies, for all $x, y, z \in A$,

$$
\begin{aligned}
& \left(1^{\prime}\right) d(\overline{0})=\overline{0}, \\
& \left(2^{\prime}\right) x \leq d(x), \\
& \left(3^{\prime}\right) \text { if }(x \rightarrow y) \vee z=\overline{1} \text { then }(d(x) \rightarrow d(y)) \vee z=\overline{1} .
\end{aligned}
$$

Analogously to the case of truth-stressers, every L-chain $\boldsymbol{A}$ can be expanded to an $\mathrm{L}_{d^{-}}$-chain by adding an arbitrary non-decreasing mapping $d: A \rightarrow A$ such that $d(\overline{0})=\overline{0}$ and $x \leq d(x)$ for every $x \in A$.

Also, since the lattice disjunction keeps satisfying the (PCP) in the expanded logic, $\mathrm{L}_{d}$ is semilinear and hence it is complete with respect to the semantics of all $\mathrm{L}_{d}$-chains. As a straightforward consequence, we have for $d$ an analogous result to Corollary 10 for $s$ :

Lemma 22. In $\mathrm{L}_{d}$ the following deductions are valid: 
(vi) $\vdash_{\mathrm{L}_{d}} d(\varphi \vee \psi) \leftrightarrow d \varphi \vee d \psi$,

(vii) $\vdash_{\mathrm{L}_{d}} d(\varphi \wedge \psi) \leftrightarrow d \varphi \wedge d \psi$.

The next theorem is easily proved in an analogous way as for truth-stressers.

Theorem 23. Let $\mathcal{L}$ be the language of a core fuzzy logic L. For every set $\Gamma \cup\{\varphi\}$ of $\mathcal{L}$-formulae, $\Gamma \vdash_{\mathrm{L}_{d}} \varphi$ iff $\Gamma \vdash_{\mathrm{L}} \varphi$.

Also, as in the case of truth-stressers, the following remarks are provable:

1. Monotonicity with respect to $\wedge$ and $\vee$ are not equivalent.

2. $\left(\mathrm{MON}_{d}\right)$ cannot be equivalently substituted by the monotonicity axioms with respect to both lattice connectives: $d(\varphi \wedge \psi) \leftrightarrow d \varphi \wedge d \psi$ and $d(\varphi \vee \psi) \leftrightarrow d \varphi \vee d \psi$.

3. The inference rule $\left(\mathrm{MON}_{d}\right)$ cannot be equivalently simplified to: from $\varphi \rightarrow \psi$ infer $d(\varphi) \rightarrow$ $d(\psi)$.

4. The inference rule $\left(\mathrm{MON}_{d}\right)$ cannot be equivalently substituted by this rule: from $\neg \varphi$ infer $\neg d(\varphi)$.

The proofs of these statements are analogous to the ones of the corresponding results for truthstressers. For 1 take the dual of the Gödel algebra of example 2, i.e. the finite non-linear Gödel algebra defined over $\{0, a, b, c, 1\}$ where 0 is the minimum, 1 is the maximum, $a$ is the only co-atom, $c \wedge b=0$, and $c \vee b=a$ and the dual truth-depresser. For 2 take the same Gödel algebra of the previous case, the filter $F=\{c, a, 1\}$ and $d$ defined by $d(1)=d(a)=d(b)=1$ and $d(c)=d(0)=0$ and proceed as in Example 3. Modifying Example 4 by taking $d(a, b)=\langle a \vee b, a \vee b\rangle$, we can prove that, in the context of truth-depressers, the rule $\left(\mathrm{MON}_{d}\right)$ cannot be substituted by simple monotonicity, i.e. we have proved 3. The same example also proves 4 .

Completeness results analogous to Theorems 3 and 4, and Corollaries 11 and 12 can be easily proved and they are summarized next.

Theorem 8. Let $\mathrm{L}$ be a core fuzzy logic, let $\mathbb{K}$ be a class of $\mathrm{L}$-chains, and let $\mathbb{K}_{d}$ be the class of the $\mathrm{L}_{d}$-chains whose $d$-free reducts are in $\mathbb{K}$.

If the language of $\mathrm{L}$ is finite we have:

- L has the $\mathrm{FSKC}$ if, and only if, $\mathrm{L}_{d}$ has the $\mathrm{FSK}_{d} \mathrm{C}$.

- In particular, $\mathrm{L}$ has the $\mathrm{FSRC}$ if, and only if, $\mathrm{L}_{d}$ has the $\mathrm{FSRC}$.

If the chains in $\mathbb{K}$ are completely ordered we have:

- $\mathrm{L}$ has the $\mathrm{SKC}$ if, and only if, $\mathrm{L}_{d}$ has the $\mathrm{SK}_{d} \mathrm{C}$.

- In particular, $\mathrm{L}_{d}$ has the $\mathrm{SRC}$ if, and only if, $\mathrm{L}$ has the $\mathrm{SRC}$.

\section{Deduction theorems and the variety problem for logics $\mathrm{L}_{d}$}

Similar results to the ones for truth-stressers given in Section 4 also hold for truth-depressers, although with some variations. The class of algebras associated to a logic $\mathrm{L}_{d}$ is a quasivariety and, like in the case of the logics of truth-stressers $\mathrm{L}_{s}$, we do not know whether these classes are always varieties or not. When $\mathrm{L}$ is a $\triangle$-fuzzy core logic, this question is clear, as in to the case 
of truth-stressers (see Section 4.2), since then the $\left(\mathrm{MON}_{d}\right)$ inference rule can be replaced by the axiom $\triangle(\varphi \rightarrow \psi) \rightarrow(d \varphi \rightarrow d \psi)$ and hence $\mathrm{L}_{d}$ is actually a $\triangle$-core fuzzy logic as well. In other cases, according to Theorem 5 , one way to prove that the quasivariety of $\mathrm{L}_{d}$-algebras is a variety is to prove that the logic $\mathrm{L}_{d}$ has either a global or local, deduction theorem. In this section we prove that this is in fact the case for two classes of logics L.

\subsection{The case of $n$-contractive axiomatic expansions of $B L$}

Observe first that, in the case of truth-depressers, the property that an L-filter is closed under $d$ has no sense since, being $d(x) \geq x$, any L-filter is closed under $d$. However, this does not mean that the $\mathrm{L}_{d}$ filters coincide with $\mathrm{L}$ filters. Take for example the same algebra as in Example 5 and $\mathrm{d}$ defined by $d(x)=x$ for all $x \neq b$ and $d(b)=a$. Then the L-filter $F=\{1, a\}$ is not an $\mathrm{L}_{d}$-filter since $b \rightarrow c=a \in F$ and $d(b) \rightarrow d(c)=a \rightarrow c=b \notin F$.

In this case we will use a different and more general way to prove that any $\operatorname{logic} \mathrm{L}_{d}$ where $\mathrm{L}$ is an $n$-contractive axiomatic expansion of BL enjoys a global deduction theorem. Recall that, given $n \geq 2$, a logic $\mathrm{L}$ is $n$-contractive if it proves the $n$-contraction law $\varphi^{n-1} \rightarrow \varphi^{n}$. For the proof of the result, we will use the following theorem of BL (it is folkore, but we include its proof for the reader's convenience):

Lemma 24. The following formula is provable in $\mathrm{BL}$ :

$$
(\varphi \rightarrow \varphi \& \varphi) \rightarrow((\varphi \wedge \psi) \rightarrow \varphi \& \psi)) .
$$

Proof. We prove it by cases, by showing the following provabilities in BL:

(i) $\vdash(\varphi \rightarrow \psi) \rightarrow(\varphi \& \varphi \rightarrow \varphi \& \psi)$

(ii) $\varphi \rightarrow \psi \vdash(\varphi \rightarrow \varphi \& \varphi) \rightarrow(\varphi \rightarrow \varphi \& \psi)$, and hence $\varphi \rightarrow \psi \vdash(\varphi \rightarrow \varphi \& \varphi) \rightarrow(\varphi \wedge \psi \rightarrow \varphi \& \psi)$

(iii) $\psi \rightarrow \varphi \vdash \psi \rightarrow(\varphi \wedge \psi)$, and hence $\psi \rightarrow \varphi \vdash \psi \rightarrow(\varphi \&(\varphi \rightarrow \psi))$

(iv) $\psi \rightarrow(\varphi \&(\varphi \rightarrow \psi)) \vdash(\varphi \rightarrow \varphi \& \varphi) \rightarrow(\psi \rightarrow(\varphi \& \varphi \&(\varphi \rightarrow \psi)))$ $\psi \rightarrow(\varphi \&(\varphi \rightarrow \psi)) \vdash(\varphi \rightarrow \varphi \& \varphi) \rightarrow(\psi \rightarrow(\varphi \&(\varphi \wedge \psi)))$ $\psi \rightarrow \varphi, \psi \rightarrow(\varphi \&(\varphi \rightarrow \psi)) \vdash(\varphi \rightarrow \varphi \& \varphi) \rightarrow(\psi \rightarrow(\psi \& \varphi))$ $\psi \rightarrow \varphi, \psi \rightarrow(\varphi \&(\varphi \rightarrow \psi)) \vdash(\varphi \rightarrow \varphi \& \varphi) \rightarrow((\varphi \wedge \psi) \rightarrow(\psi \& \varphi))$

(v) from (iii) and (iv), $\psi \rightarrow \varphi \vdash(\varphi \rightarrow \varphi \& \varphi) \rightarrow((\varphi \wedge \psi) \rightarrow(\psi \& \varphi))$

From (ii) and (v) we finally get $\vdash(\varphi \rightarrow \varphi \& \varphi) \rightarrow((\varphi \wedge \psi) \rightarrow \varphi \& \psi)$.

Notice that if $\mathrm{L}$ is $n$-contractive then $\varphi^{n-1}$ is idempotent, i.e. L proves the formula $\varphi^{n-1} \rightarrow$ $\varphi^{n-1} \& \varphi^{n-1}$, and hence L proves $\left(\varphi^{n-1} \wedge \psi\right) \rightarrow\left(\varphi^{n-1} \& \psi\right)$ as well.

Theorem 9. Let $\mathrm{L}$ be an $n$-contractive axiomatic expansion of $\mathrm{BL}$ for some $n \geq 2$. Then the logic $\mathrm{L}_{d}$ enjoys the following global deduction-detachment theorem: for every set $\Gamma \cup\{\varphi, \psi\}$ of formulae, it holds that

$$
\Gamma, \varphi \vdash_{\mathrm{L}_{d}} \psi \text { iff } \Gamma \vdash_{\mathrm{L}_{d}} \varphi^{n-1} \rightarrow \psi \text {. }
$$


Proof. Again, we only show the induction step for the inference rule: if $\Gamma \vdash_{\mathrm{L}_{d}} \delta^{n-1} \rightarrow((\varphi \rightarrow \psi) \vee \chi)$ then $\Gamma \vdash_{\mathrm{L}_{d}} \delta^{n-1} \rightarrow((d \varphi \rightarrow d \psi) \vee \chi)$. Notice that since $\mathrm{L}$ is $n$-contractive, then $\mathrm{L}_{d}$ proves $\delta^{n-1} \rightarrow \delta^{n-1} \& \delta^{n-1}$, and by Lemma $24, \mathrm{~L}_{d}$ also proves $\left(\delta^{n-1} \wedge \varphi\right) \rightarrow \delta^{n-1} \& \varphi$. Now assume $\Gamma \vdash_{\mathrm{L}_{d}} \delta^{n-1} \rightarrow((\varphi \rightarrow \psi) \vee \chi)$. By cases again, we have:

(i) $\Gamma,(\varphi \rightarrow \psi) \rightarrow \chi \vdash_{\mathrm{L}_{d}} \delta^{n-1} \rightarrow \chi$, and hence $\Gamma,(\varphi \rightarrow \psi) \rightarrow \chi \vdash_{\mathrm{L}_{d}} \delta^{n-1} \rightarrow(d \varphi \rightarrow d \psi) \vee \chi$ as well.

(ii) $\Gamma, \chi \rightarrow(\varphi \rightarrow \psi) \vdash_{\mathrm{L}_{d}} \delta^{n-1} \rightarrow(\varphi \rightarrow \psi)$, and hence $\Gamma, \chi \rightarrow(\varphi \rightarrow \psi) \vdash_{\mathrm{L}_{d}} d\left(\delta^{n-1} \& \varphi\right) \rightarrow d \psi$ as well. But, $\vdash_{\mathrm{L}_{d}}\left(\delta^{n-1} \wedge \varphi\right) \rightarrow \delta^{n-1} \& \varphi$, and thus $\vdash_{\mathrm{L}_{d}} d\left(\delta^{n-1} \wedge \varphi\right) \rightarrow d\left(\delta^{n-1} \& \varphi\right)$, and taking into account that $\vdash_{\mathrm{L}_{d}} d\left(\delta^{n-1} \wedge \varphi\right) \leftrightarrow d\left(\delta^{n-1}\right) \wedge d \varphi$, hence we finally have $\Gamma, \chi \rightarrow(\varphi \rightarrow \psi) \vdash_{\mathrm{L}_{d}}$ $\delta^{n-1} \rightarrow(d \varphi \rightarrow d \psi)$.

Finally, from (i) and (ii) we have $\Gamma \vdash_{\mathrm{L}_{d}} \delta^{n-1} \rightarrow((d \varphi \rightarrow d \psi) \vee \chi)$.

Observe that every finite BL-chain with $n$ elements satisfy the $n$-contraction identity $\left(x^{n-1}=\right.$ $\left.x^{n}\right)$ and thus, in particular, if $\mathrm{L}$ is the logic of a finite BL-chain, the logic $\mathrm{L}_{d}$ enjoys the global deduction-detachment theorem.

The last theorem, together with Theorem 5, immediately yields the following corollary.

Corollary 25. Let $\mathrm{L}$ be an n-contractive axiomatic expansion of BL. The quasivariety associated to the logic $\mathrm{L}_{d}$ is a variety.

In particular, if $\mathrm{L}$ is the logic of a finite BL-chain, the quasivariety associated to $\mathrm{L}_{d}$ is a variety.

\subsection{Logics satisfying the modal axioms $K$ for the truth-depressers}

In this section we consider axiomatic extensions of a logic $\mathrm{L}_{d}$ with the following two modal-like axioms used by Vychodil [38] to define two systems for truth-depressers as expansions of BL:

$(\mathrm{ST} 5) d(\varphi \rightarrow \psi) \rightarrow(d \varphi \rightarrow d \psi)$

(ST6) $(\varphi \rightarrow \psi) \rightarrow(d \varphi \rightarrow d \psi)$

Let us define the logics $\mathrm{L}_{d-\mathrm{I}}$ and $\mathrm{L}_{d-\mathrm{II}}$ as the axiomatic extensions of $\mathrm{L}_{d}$ with the axioms (ST5) and (ST6) respectively.

A first obvious observation is that the system $\mathrm{L}_{d-\mathrm{I}}$ is stronger than $\mathrm{L}_{d-\mathrm{II}}$ since the axiom (ST6) is derivable in the system $\mathrm{L}_{d-\mathrm{II}}$.

A second observation is that the rule $\left(\mathrm{MON}_{d}\right)$ is superfluous in both systems $\mathrm{L}_{d-\mathrm{I}}$ and $\mathrm{L}_{d-\mathrm{II}}$. Indeed, it is very easy to check that in both systems one can prove the following formula

$$
((\varphi \rightarrow \psi) \vee \chi) \rightarrow((d \varphi \rightarrow d \psi) \vee \chi)
$$

without using the rule $\left(\mathrm{MON}_{d}\right)$, just using the axioms (ST1) and (ST5) in the case of $\mathrm{L}_{d-\mathrm{I}}$, and using axiom (ST6) in the case of $\mathrm{L}_{d \text {-II }}$. Therefore, both logics $\mathrm{L}_{d \text {-I }}$ and $\mathrm{L}_{d \text {-II }}$ are indeed axiomatic expansions of $\mathrm{L}$, and hence we have the following results for free.

Lemma 26. Let $\mathrm{L}$ be any core fuzzy logic. Then both $\mathrm{L}_{d-I}$ and $\mathrm{L}_{d-I I}$ are core fuzzy logics too, and thus: 
- they are complete with respect to their corresponding classes of chains,

- they satisfy the same local/global deduction-detachment theorem as $\mathrm{L}$, and

- the quasivarieties associated to $\mathrm{L}_{d-I}$ and $\mathrm{L}_{d-I I}$ are in fact varieties.

The following lemma shows that if $\mathrm{L}$ is a core fuzzy logic expanding IMTL, the involutive extension of MTL, in particular Lukasiewicz logic or Nilpotent Minimum logic, then $\mathrm{L}_{d \text {-II }}$, and hence $\mathrm{L}_{d \text {-I }}$ as well, collapses with $\mathrm{L}$ itself.

Lemma 27. Let $\mathrm{L}$ be any core fuzzy logic expanding IMTL. Then both $\mathrm{L}_{d-I}$ and $\mathrm{L}_{d-I I}$ prove the formula

$$
\varphi \leftrightarrow d \varphi
$$

Proof. It is enough to show that $\mathrm{L}_{d \text {-II }}$ proves the formula $d \varphi \rightarrow \varphi$. But this is easy since taking taking $\psi$ as $\overline{0}$ in axiom (ST6), one gets $\neg \varphi \rightarrow(d \varphi \rightarrow d \overline{0})$. Now, using (ST2), that is simply $d \overline{0} \rightarrow \overline{0}$, we can prove $\neg \varphi \rightarrow(d \varphi \rightarrow \overline{0})$, i.e. $\neg \varphi \rightarrow \neg d \varphi$. Finally, recalling that in IMTL the contraposition law $(\varphi \rightarrow \psi) \rightarrow(\neg \psi \rightarrow \neg \varphi)$ holds, one can prove $d \varphi \rightarrow \varphi$.

In particular, this result shows that, for WNM logics, expansions with truth-depressers behave much differently than those with truth-stressers. Indeed, for truth-stressers we have proved that if $\mathrm{L}$ is the logic of a WNM-chain, the logics $\mathrm{L}_{s}$ and $\mathrm{L}_{s K}$ are the same. However, according to the above result, $\mathrm{NM}_{d I}=\mathrm{NM}_{d I I}=\mathrm{NM}$, while this is clearly not true for $\mathrm{NM}_{d}$. Indeed axioms (ST5) and (ST6) are not sound over the standard NM-chain. For instance, take the truth-depresser defined by $d(x)=0.5$ if $x \in(0,0.2), d(x)=0.6$ if $x \in[0.2,0.6]$ and $d(x)=x$ otherwise. Then ${ }^{14}$ $0.2 \rightarrow 0.1=0.8=d(0.2 \rightarrow 0.1)$ and $d(0.2) \rightarrow d(0.1)=0.6 \rightarrow 0.5=0.5$, therefore $(0.2 \rightarrow 0.1) \rightarrow$ $(d(0.2) \rightarrow d(0.1))=0.5<1$. This shows that (ST6) is not sound. The same holds true for (ST5) just by replacing $(0.2 \rightarrow 0.1)$ by $d(0.2 \rightarrow 0.1)$ in the expression above, because the two expressions have the same value.

\section{Some further topics}

\subsection{Logics with both a truth-stresser and a truth-depresser}

Given a core fuzzy logic $\mathrm{L}$, let us define the $\operatorname{logic} \mathrm{L}_{s, d}$ as the expansion of $\mathrm{L}_{s}$ with a new unary connective $d$ together with the axioms (STL1) and (STL2), and the inference rule $\left(\mathrm{MON}_{d}\right)$.

The algebraic semantics of $\mathrm{L}_{s, d}$ is given by $\mathrm{L}_{s, d}$-algebras, which are defined in the natural way as expansions of $\mathrm{L}_{s}$-algebras with a new unary operation $d$ satisfying the equations corresponding to axioms (STL1) and (STL2) and the quasiequation corresponding to $\left(\mathrm{MON}_{d}\right)$.

Actually, since in $\mathrm{L}_{s, d}$ the stressers $s$ and $d$ are independent from each other, most properties we have discussed in the previous sections easily extend to $\mathrm{L}_{s, d}$; in particular, chain-completeness and real completeness results directly extend from $\mathrm{L}_{s}$ and $\mathrm{L}_{d}$ to $\mathrm{L}_{s, d}$.

Corollary 28. Let $\mathrm{L}$ be a core fuzzy logic. Then the following properties hold for $\mathrm{L}_{s, d}$ :

- $\mathrm{L}_{s, d}$ is complete with respect to the class of $\mathrm{L}_{s, d}$-chains.

\footnotetext{
${ }^{14}$ Recall that the implication operation in the standard NM-chain is defined as $x \rightarrow y=1$ if $x \leq y$, and $x \rightarrow y=$ $\max \{1-x, y\}$ otherwise.
} 
- $\mathrm{L}_{s, d}$ is a conservative expansion of $\mathrm{L}_{s}, \mathrm{~L}_{d}$ and $\mathrm{L}$.

Corollary 29. Let $\mathrm{L}$ be a core fuzzy logic, let $\mathbb{K}$ be a class of $\mathrm{L}$-chains, and let $\mathbb{K}_{s, d}$ be the class of the $\mathrm{L}_{s, d^{-}}$-chains whose $\{s, d\}$-free reducts are in $\mathbb{K}$.

If the language of $\mathrm{L}$ is finite we have:

- $\mathrm{L}$ has the $\mathrm{FSKC}$ if, and only if, $\mathrm{L}_{s, d}$ has the $\mathrm{FSK}_{s, d} \mathrm{C}$.

- In particular, $\mathrm{L}$ has the $\mathrm{FSRC}$ if, and only if, $\mathrm{L}_{s, d}$ has the $\mathrm{FSRC}$.

If the chains in $\mathbb{K}$ are completely ordered we have:

- $\mathrm{L}$ has the $\mathrm{SKC}$ if, and only if, $\mathrm{L}_{s, d}$ has the $\mathrm{SK}_{s, d} \mathrm{C}$.

- In particular, $\mathrm{L}_{s, d}$ has the $\mathrm{SRC}$ if, and only if, $\mathrm{L}$ has the $\mathrm{SRC}$.

One can also consider the additional axiom (in fact two axioms):

(Cont) $d s \varphi \leftrightarrow s d \varphi \leftrightarrow \varphi$

Let us denote the expansion of the logic $\mathrm{L}_{s, d}$ with these axioms as $\mathrm{L}_{s, d}^{\text {cont }}$. It is easy to check that the result of Corollary 29 regarding FSRC remains true, i.e. L has the FSRC if, and only if, $\mathrm{L}_{s, d}^{\text {cont }}$ has the FSRC. Moreover it is obvious that the corresponding equations over a real chain imply that the functions interpreting hedges have to be continuous (take into account that $d(s(x))=s(d(x))=x$ implies that $s$ and $d$ are bijective and $d=s^{-1}$ ). As a consequence, one can indeed consider $\mathrm{L}_{s, d}^{\text {cont }}$ as the logic (expansion of $\mathrm{L}$ ) of continuous stressing and depressing hedges.

In Vychodil's logic $\mathrm{BL}_{v t, s t}$, the author also considered the following axioms (relating stressers and depressers),

(ST2) $d \varphi \rightarrow \neg s \neg \varphi$,

(ST3) $s(\varphi \rightarrow \psi) \rightarrow(d \varphi \rightarrow d \psi)$

but we will not further explore these kind of expansions (actually axiomatic extensions of our $\mathrm{L}_{s, d}$ logics) here since it is not clear how to justify these type of axioms relating truth stressers and depressers. Semantically (ST2) gives an upper bound of the depresser obtained by dualitization of the stresser, while (ST3) is a modification of the axiom K. However, in a similar way, we could define other axioms with different meanings and we have no argumentation to give priority to one of them. Moreover, for these expansions, it is not clear how to obtain real completeness.

\subsection{Expansions with truth-constants}

The logics $\mathrm{L}_{s}$ and $\mathrm{L}_{d}$ axiomatize respectively the whole classes of stressing and depressing hedges on $[0,1]$. However, sometimes we are interested on reasoning with a particular hedge or a particular family of hedges, for example, when one wants to build a fuzzy description language able to manage linguistic modifiers as proposed in [37,36]. If $\mathrm{L}$ is the logic of a suitable t-norm $*$, one can resort to expanding the logic with truth-constants and (partially) specify the hedge by means of book-keeping axioms for the hedge.

Let $\mathrm{L}$ be the logic of a left-continuous t-norm $*$, i.e. such that $\mathrm{L}$ is complete with respect to the standard algebra $[0,1]_{*}=\left\langle[0,1], *, \Rightarrow_{*}, \min , \max , 0,1\right\rangle$, where $\Rightarrow_{*}$ is the residuum of $*$ and expand it with truth-constants from a countable subalgebra $C \subseteq[0,1]_{*}$. We also expand it with the projection 
operator $\triangle$, obtaining the logic $\mathrm{L}_{* \Delta}(\boldsymbol{C})$. We will assume that such logic retains the finite strong completeness with respect to its standard algebra $\left\langle[0,1], *, \Rightarrow_{*}, \min , \max , 0,1, \triangle,\{r \mid r \in C\}\right\rangle$. This is always the case when $*$ is a continuous t-norm decomposable as an ordinal sum of finitely-many basic components such that $\boldsymbol{C}$ has elements in the interior of each component (see [15, Theorem 2.8.3.]) or when $*$ is a WNM t-norm satisfying analogous properties (see [16, Theorem 32]).

As an example, consider the $\operatorname{logic} \mathrm{L}_{\triangle, s}(\mathrm{Q})$, i.e. the expansion of the logic $\mathrm{L}_{s}$ with rational truth-constants and with the $\triangle$ operator. Let us fix a truth-stressing hedge $f:[0,1] \rightarrow[0,1]$ that is closed over the rationals. Axioms and rules of $\mathrm{L}_{\triangle, s}^{f}(\mathrm{Q})$ are those of $\mathrm{L}_{\triangle, s}$ plus the following book-keeping axioms:

(Book-*) $\quad \bar{r} \& \bar{q} \leftrightarrow \bar{r} * q$ for every $r, q \in[0,1] \cap \mathrm{Q}$,

$\left(\right.$ Book- $\left.\Rightarrow_{*}\right) \quad(\bar{r} \rightarrow \bar{q}) \leftrightarrow \bar{r} \Rightarrow_{*} q$ for every $r, q \in[0,1] \cap \mathrm{Q}$,

(Book- $\triangle) \quad \triangle(\bar{r}) \leftrightarrow \overline{\triangle(r)}$, for each $r \in[0,1] \cap \mathrm{Q}$,

(Book- $f) \quad s(\bar{r}) \leftrightarrow \overline{f(r)}$, for each $r \in[0,1] \cap \mathrm{Q}$.

The algebraic semantics is given by the class of $\mathrm{L}_{\triangle, s}^{f}(\mathrm{Q})$-algebras which are structures $\boldsymbol{A}=$ $\left\langle A, \&, \rightarrow, \wedge, \vee, \triangle, s,\left\{\bar{r}^{\boldsymbol{A}}\right\}_{r \in[0,1] \cap \mathrm{Q}}\right\rangle$ where $\langle A, \&, \rightarrow, \wedge, \vee, \triangle, s, \overline{0}, \overline{1}\rangle$ is an $\mathrm{L}_{\triangle, s}$-algebra and for each rational $r \in[0,1], \bar{r}^{\boldsymbol{A}}$ is a 0 -ary operation (i.e. a value in $A$ ), satisfying the following conditions:

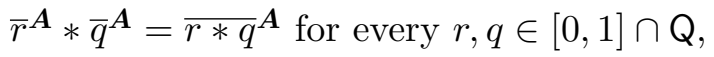

$$
\begin{aligned}
& \bar{r}^{\boldsymbol{A}} \Rightarrow_{*} \bar{q}^{\boldsymbol{A}}=\bar{r}_{{ }_{*} q^{\boldsymbol{A}}} \text { for every } r, q \in[0,1] \cap \mathrm{Q}, \\
& \triangle^{\boldsymbol{A}}\left(\bar{r}^{\boldsymbol{A}}\right)=\overline{\triangle(r)}^{\boldsymbol{A}} \text {, for each } r \in[0,1] \cap \mathrm{Q}, \\
& s\left(\bar{r}^{\boldsymbol{A}}\right)=\overline{f(r)}^{\boldsymbol{A}} \text {, for each } r \in[0,1] \cap \mathbf{Q} \text {. }
\end{aligned}
$$

Since $\mathrm{L}_{\triangle, s}^{f}(\mathrm{Q})$ is an axiomatic expansion of $\mathrm{L}_{\triangle, s}$, it is also a $\triangle$-core fuzzy logic, the class of $\mathrm{L}_{\triangle, s}^{f}\left(\mathrm{Q}\right.$ )-algebras is a variety, and $\mathrm{L}_{\triangle, s}^{f}(\mathrm{Q})$ is (strongly) complete with respect to the class of $\mathrm{L}_{\triangle, s}^{f}(\mathrm{Q})$-chains. Moreover, we can prove the following real completeness with respect to the intended semantics.

Theorem 10. $\mathrm{L}_{\triangle, s}^{f}(\mathrm{Q})$ is finite strong complete with respect to the standard $\mathrm{L}_{\triangle, s}^{f}(\mathrm{Q})$-algebra $[0,1]_{\triangle, s}^{f}=\left\langle[0,1], *, \Rightarrow_{*}, \min , \max , \triangle, f,\{r\}_{r \in[0,1] \cap Q}\right\rangle$.

Proof. Assume that $\Gamma \nvdash_{\mathrm{L}_{\triangle, s}^{f}(\mathrm{Q})} \varphi$, for some finite set of formulae $\Gamma \cup\{\varphi\}$. Then there is an $\mathrm{L}_{\triangle, s}^{f}(\mathrm{Q})$-chain $\boldsymbol{A}$ and an $\boldsymbol{A}$-evaluation $e$ such that $e[\Gamma] \subseteq\left\{\overline{1}^{\boldsymbol{A}}\right\}$ while $e(\varphi)<\overline{1}^{\boldsymbol{A}}$. Let $X \subseteq A$ be the finite set of images by $e$ of all subformulae of $\Gamma \cup\{\varphi\}$. Let $\boldsymbol{A}^{-}$be the $\mathrm{L}_{\triangle}(\mathrm{Q})$-reduct of $\boldsymbol{A}$. Since $\mathrm{L}_{\triangle}(\mathrm{Q})$ is finite strong complete with respect to the standard $\mathrm{L}_{\triangle}(\mathrm{Q})$-algebra, there is a partial embedding $h$ from $\boldsymbol{A}^{-}$into $[0,1]$ respecting all operations in $X$ and such that $h\left(\bar{r}^{\boldsymbol{A}}\right)=r$ for all $\bar{r}$ appearing in $\Gamma \cup\{\varphi\}$. In particular, since $s\left(\bar{r}^{\boldsymbol{A}}\right)=\overline{f(r)}^{\boldsymbol{A}}$, if both $\bar{r}^{\boldsymbol{A}}$ and $s\left(\bar{r}^{\boldsymbol{A}}\right)$ are in $X$ then $h\left(s\left(\bar{r}^{\boldsymbol{A}}\right)\right)=h\left(\overline{f(r)}^{\boldsymbol{A}}\right)=f(r)$. Therefore, $h$ is actually a partial embedding from $\boldsymbol{A}$ into $[0,1]_{\triangle, s}^{f}$, and hence $e^{\prime}=h \circ e$ is an evaluation on $[0,1]_{\triangle, s}^{f}$ such that $e^{\prime}[\Gamma] \subseteq\{1\}$ and $e^{\prime}(\varphi)<1$. 


\subsection{First-order logics with hedges}

In this final subsection we will consider first-order fuzzy logics expanded with hedges. First we need to recall the usual presentation of first-order formalisms for core fuzzy logics.

Given a propositional core fuzzy logic $\mathrm{L}$, the language $\mathcal{P} \mathcal{L}$ of $\mathrm{L} \forall$ is built in the standard classical way from the propositional language $\mathcal{L}$ of $\mathrm{L}$ by enlarging it with a set of predicate symbols Pred and a set of function symbols Funct and a set of object variables Var, together with the two classical quantifiers $\forall$ and $\exists$. The set of terms Term is the minimum set containing the elements of $V a r$ and closed under the functions. The atomic formulae are expressions of the form $P\left(t^{1}, \ldots, t^{n}\right)$, where $P \in$ Pred and $t^{1}, \ldots, t^{n} \in$ Term. The set of all formulae is obtained by closing the set of atomic formulae under combination by propositional connectives and quantification, i.e. if $\varphi$ is a formula and $x$ is an object variable, then $(\forall x) \varphi$ and $(\exists x) \varphi$ are formulae as well.

In first-order core fuzzy logics it is usual to restrict the semantics to chains only. Given an Lchain $\boldsymbol{A}$, an $\boldsymbol{A}$-structure is $\mathbf{M}=\left\langle M,\left\langle P_{\mathbf{M}}\right\rangle_{P \in \text { Pred }},\left\langle f_{\mathbf{M}}\right\rangle_{f \in F u n c t}\right\rangle$ where $M \neq \emptyset, f_{\mathbf{M}}: M^{\operatorname{ar}(f)} \rightarrow M$, and $P_{\mathbf{M}}: M^{\operatorname{ar}(P)} \rightarrow A$ for each $f \in$ Funct and $P \in$ Pred (where ar is the function that gives the arity of function and predicate symbols). For each evaluation of variables $v: \operatorname{Var} \rightarrow M$, the interpretation of a $t \in T e r m$, denoted $t_{\mathbf{M}, v}$, is defined as in classical first-order logic. The truth-value $\|\varphi\|_{\mathbf{M}, v}^{\boldsymbol{A}}$ of a formula is defined inductively from

$$
\left\|P\left(t^{1}, \ldots, t^{n}\right)\right\|_{\mathbf{M}, v}^{\boldsymbol{A}}=P_{\mathbf{M}}\left(t_{\mathbf{M}, v}^{1}, \ldots, t_{\mathbf{M}, v}^{n}\right),
$$

taking into account that the value commutes with connectives, and defining

$$
\begin{aligned}
& \|(\forall x) \varphi\|_{\mathbf{M}, v}^{\boldsymbol{A}}=\inf \left\{\|\varphi\|_{\mathbf{M}, v^{\prime}}^{\boldsymbol{A}} \mid v(y)=v^{\prime}(y) \text { for all variables } y, \text { except } x\right\} \\
& \|(\exists x) \varphi\|_{\mathbf{M}, v}^{\boldsymbol{A}}=\sup \left\{\|\varphi\|_{\mathbf{M}, v^{\prime}}^{\boldsymbol{A}} \mid v(y)=v^{\prime}(y) \text { for all variables } y, \text { except } x\right\}
\end{aligned}
$$

if the infimum and supremum exist in $\boldsymbol{A}$, otherwise the truth-value(s) remain undefined. An $\boldsymbol{A}$ structure $\mathbf{M}$ is called safe if all infs and sups needed for the definition of the truth-value of any formula exist in $\boldsymbol{A}$. Then the truth-value of a formula $\varphi$ in a safe $\boldsymbol{A}$-structure $\mathbf{M}$ is just

$$
\|\varphi\|_{\mathbf{M}}^{\boldsymbol{A}}=\inf \left\{\|\varphi\|_{\mathbf{M}, v}^{\boldsymbol{A}} \mid v: \operatorname{Var} \rightarrow M\right\} .
$$

When $\|\varphi\|_{\mathbf{M}}^{\boldsymbol{A}}=1$ for a safe $\boldsymbol{A}$-structure $\mathbf{M}$, the pair $\langle\mathbf{M}, \boldsymbol{A}\rangle$ is said to be a model for $\varphi$, written $\langle\mathbf{M}, \boldsymbol{A}\rangle \models \varphi$.

The axioms for $\mathrm{L} \forall$ are obtained from those of $\mathrm{L}$ by substitution of propositional variables with formulae of $\mathcal{P} \mathcal{L}$ plus the following axioms for quantifiers:

$(\forall 1) \quad(\forall x) \varphi(x) \rightarrow \varphi(t)(t$ substitutable for $x$ in $\varphi(x))$

$(\exists 1) \quad \varphi(t) \rightarrow(\exists x) \varphi(x)(t$ substitutable for $x$ in $\varphi(x))$

$(\forall 2) \quad(\forall x)(\nu \rightarrow \varphi) \rightarrow(\nu \rightarrow(\forall x) \varphi)(x$ not free in $\nu)$

$(\exists 2) \quad(\forall x)(\varphi \rightarrow \nu) \rightarrow((\exists x) \varphi \rightarrow \nu)(x$ not free in $\nu)$

$(\forall 3) \quad(\forall x)(\varphi \vee \nu) \rightarrow((\forall x) \varphi \vee \nu)(x$ not free in $\nu)$

The rules of inference of $L \forall$ are modus ponens and generalization: from $\varphi$ infer $(\forall x) \varphi$.

Theorem 11. For any first-order core fuzzy logic $\mathrm{L} \forall$, any set of sentences $T$ and any formula $\varphi$, it holds that $T \vdash_{\mathrm{L} \forall} \varphi$ iff $\langle\mathbf{M}, \boldsymbol{A}\rangle \models \varphi$ for each model $\langle\mathbf{M}, \boldsymbol{A}\rangle$ of $T$ with $\boldsymbol{A}$ being a countable L-chain. 
The properties $\mathrm{S} \mathbb{K C}, \mathrm{FS} \mathbb{K C}$ and $\mathbb{K C}$ are defined analogously as in the propositional case. Observe that the previous theorem says that every first-order core fuzzy logic enjoys the SKC when $\mathbb{K}$ is the class of all countable chains. A usual way to prove $\mathrm{S} \mathbb{K} C$ consists on showing that every non-trivial countable L-chain can be $\sigma$-embedded (i.e. with an embedding which preserves existing suprema and infima) into some chain from $\mathbb{K}$. In this case we say that $\mathrm{L}$ has the $\mathbb{K}-\sigma$ embedding property. As proved in [9] this is a sufficient, but in general not necessary, condition for the SKC. This method has been used to prove strong real completeness for first-order versions of a number of important fuzzy logics such as MTL, SMTL, IMTL, G, NM, and WNM. Others have been shown to lack all real completeness properties as a consequence of the studies on the arithmetical complexity of the set of standard tautologies as in the case of $\mathrm{E}, \Pi, \mathrm{BL}$, and SBL (see e.g. [24]). Moreover, it is also proved in [9] that any completeness property of a first-order logic implies the validity of the same completeness property in the underlying propositional logic.

Given a first-order core fuzzy logic $\mathrm{L} \forall$, let $\mathrm{L}_{s} \forall$ be the expansion of $\mathrm{L} \forall$ with a unary symbol $s$, the axioms (VTL1), (VTL2), and the rule $\left(\mathrm{MON}_{s}\right)$. The same logic could be obtained by considering $\mathrm{L}_{s}$ and extending it to the first-order level in the analogous way as in core fuzzy logics.

Proposition 30. $\mathrm{L}_{s} \forall$ is a conservative expansion of $\mathrm{L} \forall$.

Proof. Let $T \cup\{\varphi\}$ be a set of first-order formulae in the language without $s$ and assume that $T \nvdash_{\mathrm{L} \forall} \varphi$. Then there exists L-chain $\boldsymbol{A}$ a model $\langle\mathbf{M}, \boldsymbol{A}\rangle$ of $T$ such that $\langle\mathbf{M}, \boldsymbol{A}\rangle \not \forall \varphi$. We expand $\boldsymbol{A}$ to an $\mathrm{L}_{s}$-chain $\boldsymbol{A}^{\prime}$ where the stresser symbol is interpreted as the $\triangle$ function. $\left\langle\mathbf{M}, \boldsymbol{A}^{\prime}\right\rangle$ is still safe, is a model of $T$ and $\langle\mathbf{M}, \boldsymbol{A}\rangle \not \forall \varphi$, hence $T \nvdash_{\mathrm{L}_{s} \forall} \varphi$.

We can now consider the issue of standard completeness for these logics.

Theorem 12. Let $\mathrm{L}$ be a core fuzzy logic, $\mathbb{K}$ a class of $\mathrm{L}$-chains, and $\mathbb{K}_{s}$ the class of $\mathrm{L}_{s}$-chains whose $s$-free reducts are in $\mathbb{K}$.

1. If $\mathrm{L}$ has the $\mathbb{K}-\sigma$-embedding property and all the members of $\mathbb{K}$ are completely ordered, then $\mathrm{L}_{s} \forall$ has the $\mathrm{SK}_{s} \mathrm{C}$.

2. If $\mathrm{L}$ does not have the $\mathbb{K}$-embedding property, then $\mathrm{L}_{s} \forall$ does not have the $\mathrm{SK}_{s} \mathrm{C}$.

Proof. The first item relies on the proof of Theorem 4. Indeed assume that L has the $\mathbb{K}-\sigma$ embedding property and all the members of $\mathbb{K}$ are completely ordered and take any countable $\mathrm{L}_{s}$-chain $\boldsymbol{A}$. We know that its $s$-free reduct is $\sigma$-embeddable into a chain $\boldsymbol{B} \in \mathbb{K}$. Then the construction used in the proof of Theorem 4 allows to define a hedge function in such a way that we obtain that $\boldsymbol{B} \sigma$-embeddable into a expasnion of $\boldsymbol{B}$ in $\mathbb{K}_{s}$. As for the second item, if $\mathrm{L}$ does not have the $\mathbb{K}$-embedding property, then $\mathrm{L} \forall$ does not enjoy the $\mathrm{S} \mathbb{K} C$ and hence, since $\mathrm{L}_{s} \forall$ is a conservative expansion of $\mathrm{L} \forall, \mathrm{L}_{s} \forall$ does not have the $\mathrm{SK}_{s} \mathrm{C}$ either.

Observe that if we only know that $\mathrm{L}$ has the $\mathbb{K}$-embedding property, we cannot conclude anything, at least in general, regarding $\mathrm{SK}_{s} \mathrm{C}$ of $\mathrm{L}_{s} \forall$.

Finally, notice that the case of first-order logics expanded with a connective for depressing hedges is completely analogous: the logic can be defined by means of (STL1), (STL2) and (MON $)_{\text {), }}$ and we obtain analogous results. 


\section{Related work}

As we have mentioned before, there are two main references when talking about the formalisation of truth-stressing hedges within the framework of mathematical fuzzy logic. The first one is Hájek's paper [21] where he axiomatizes over BL a logic for the hedge very true, and the second one is the paper by Vychodil [38] where he extends Hájek's analysis to truth-depressing hedges. A further relevant further study of logics with truth-stressers can be found in the paper by Ciabattoni et al. [7], that makes significant contributions in various aspects. The authors basically consider expansions of MTL with a unary modality (i.e. a unary operator that satisfies axiom $\mathrm{K}$ and the necessitation rule), they consider three possible additional axioms to be added to Hájek's axiomatics, and they develop proof systems for the new logics and study their algebraic and completeness properties. Given a logic L that is an extension of MTL, they consider the following logics:

$$
\begin{aligned}
\mathrm{L}_{-} \mathrm{KT}^{r} & =\mathrm{L}+(\mathrm{VT} 1)+(\mathrm{VT} 2)+(\mathrm{VT} 3)+(\mathrm{NEC}) \\
\mathrm{L}-\mathrm{S} 4^{r} & =\mathrm{L}-\mathrm{KT}^{r}+(\mathrm{VT} 4) s \varphi \rightarrow s(s \varphi)
\end{aligned}
$$

Axiom (VT4), together with axiom (VT1), forces the truth-stressing hedges to be closed over their image, i.e. $s \varphi$ has to be equivalent to $s(s \varphi)$ (hence $s$ becomes a closure operator like in some previous works; see [23], for instance).

Notice that Hájek's logic $\mathrm{BL}_{v t}$ (which we have called $\mathrm{BL}_{s K}$ in this paper) is nothing but the logic BL-KT ${ }^{r}$. Moreover, Ciabattoni et al. prove in [7] standard completeness of the L-S4 ${ }^{r}$ logics for different choices for L, namely MTL, SMTL, $\mathrm{C}_{n}$ MTL, IMTL, and $\mathrm{C}_{n}$ IMTL. Finally, observe that after adding the axiom $s \varphi \vee \neg s \varphi$ to L-KT ${ }^{r}, s$ turns to be equivalent to the projection connective $\triangle$.

Other papers dealing with particular types of truth-stressers are:

- The paper [23], a pioneering work in the setting of truth-stressing hedges, which proves that the Yashin strong future tense operator can be interpreted, in our framework, as a hedge over $\mathrm{G}$ that is a closure operator and satisfies axiom $\mathrm{K}$.

- The paper [22], which defines the logical system $\mathrm{BL}_{L U}^{!}$obtained by adding two unary connectives, $\mathrm{L}$ and $\mathrm{U}$, (for truth stresser and depresser) to $\mathrm{BL}_{\triangle}$ that are required to be idempotent with respect to the monoidal operation, among other technical properties. The paper contains an interesting result about the undecidability of $*$-tautologies.

- In the paper [25] the authors introduce in BL $\forall$ a new unary connective $A t$, interpreted as almost true, in order to analyze the sorites paradox in the setting of mathematical fuzzy logic. It turns out that the axioms proposed for this new connective are (STL1) together with

$$
(\varphi \rightarrow \psi) \rightarrow(\text { At } \varphi \rightarrow \text { At } \psi)
$$

which is actually axiom (ST6), stronger than (MON). However, the axiom (STL2) is not required.

- The paper [30] studies the system obtained by adding to a fuzzy logic L a unary connective called storage operator which has some analogies with Girard's exponentials and behaves as an idempotent truth-stresser closed over its image (it is in fact an interior operator). 
- Finally, we mention the papers $[2,18,3]$ where the authors study expansions of Gödel logic with a monotone unary operator $\circ$ interpreted by monotone functions $f:[0,1] \rightarrow[0,1]$ satisfying, among other conditions, $f(1)=1$. So, although they are very close to truthhedges, in general they are not necessarily requested to satisfy the other boundary condition $f(0)=0$. However, in [18], Fasching implicity considers particular logics that indeed can be regarded as a proper logic of truth stressers: indeed, he considers the expansion of Gödel logic with $\circ$ together with the axioms $\circ(\varphi \rightarrow \psi) \leftrightarrow(\circ \varphi \rightarrow \circ \psi)^{15}$ and the rule of necessitation for $\circ$. Let us call this logic $\mathrm{G}_{\circ}$. Then, from [18, Corollaries 4.1(a) and 4.2(b)] it can readily be seen that: (i) $G_{\circ}$ together with the axiom $\circ \varphi \rightarrow \varphi$ is complete with respect to the class of standard Gödel chains expanded with strictly increasing truth-stressing hedges, and (ii) $\mathrm{G}_{\circ}$ together with the axioms $\varphi \rightarrow \circ \varphi$ and $\circ \overline{0} \rightarrow \overline{0}$ is complete with respect to the class of standard Gödel chains expanded with strictly increasing truth-depressing hedges.

Despite the undoubtable theoretical interest of these papers, hedge functions that are either closure operators, satisfy axiom K, or are idempotent, have a quite limited behavior and can account only for some very special cases of truth-stressing hedges.

Finally, it must be mentioned that, besides the axiomatic approach followed in this paper and in all the related articles we have just discussed, logics with hedges have also received an extensive treatment in the interesting formalization of evaluative linguistic expressions developed in the framework of fuzzy type theory by Vilém Novák, see e.g. [33] and Chapter 6 of [34]. Indeed, the intuition of Novák's modelling of hedges that goes back to [32] was, as pointed out by Lakoff in [28], that the effect of hedges like very in an expression like very small carries out a shifting of the whole membership function of the fuzzy set small, in the sense of a shortening of the kernel of the fuzzy set. This may be accomplished by truth-hedges $h:[0,1] \rightarrow[0,1]$ such that $h(x)=1$ if $x$ is greater than a given threshold $a$, and $h(x)=0$ for $x$ smaller than another given threshold $b$, with $0<b<a<1$. These classes of hedges are neither subdiagonal nor superdiagonal, and hence they fall out of the scope of the goals of the current paper. The question of whether these classes of truth-hedges admit a similar logical treatment than those considered in this paper (subdiagonal and superdiagonal) remains as an interesting matter of future research.

\section{Acknowledgments}

The authors are indebted to the anonymous referees for their comments that have helped improving the paper. The authors acknowledge partial support of the MICINN projects TASSAT (TIN201020967-C04-01) and ARINF (TIN2009-14704-C03-03), and the FP7-PEOPLE-2009-IRSES project MaToMUVI (PIRSES-GA-2009-247584). Carles Noguera also acknowledges support of the research contract "Juan de la Cierva" JCI-2009-05453.

\section{References}

[1] M. Baaz. Infinite-valued Gödel logic with 0-1-projections and relativisations. In P. Hájek, editor, Gödel'96: Logical Foundations of Mathematics, Computer Science, and Physics, volume 6 of Lecture Notes in Logic, pages 23-33. Springer-Verlag, Brno, 1996.

[2] M. Baaz and O. Fasching. Gödel logics with monotone operators. Fuzzy Sets and Systems, 197:3-13, 2012.

[3] M. Baaz and O. Fasching. Monotone operators on Gödel logic. Archive for Mathematical Logic, to appear.

\footnotetext{
${ }^{15}$ Notice that this axiom is stronger than axiom $\mathrm{K}$.
} 
[4] L. Běhounek, P. Cintula, and P. Hájek. Introduction to mathematical fuzzy logic. In P. Cintula, P. Hájek, and C. Noguera, editors, Handbook of Mathematical Fuzzy Logic - Volume 1, volume 37 of Studies in Logic, Mathematical Logic and Foundations. 1-101, London, 2011.

[5] W. J. Blok and D. L. Pigozzi. Algebraizable Logics, volume 396 of Memoirs of the American Mathematical Society. American Mathematical Society, Providence, RI, 1989.

[6] P. Brown and S. C. Levinson. Politeness: Some universals in language usage. Cambridge University Press, 1987.

[7] A. Ciabattoni, G. Metcalfe, and F. Montagna. Algebraic and proof-theoretic characterizations of truth stressers for MTL and its extensions. Fuzzy Sets and Systems, 161(3):369-389, 2010.

[8] R. Cignoli, F. Esteva, L. Godo, and A. Torrens. Basic fuzzy logic is the logic of continuous t-norms and their residua. Soft Computing, 4(2):106-112, 2000.

[9] P. Cintula, F. Esteva, J. Gispert, L. Godo, F. Montagna, and C. Noguera. Distinguished algebraic semantics for t-norm based fuzzy logics: Methods and algebraic equivalencies. Annals of Pure and Applied Logic, 160(1):53-81, 2009.

[10] P. Cintula and C. Noguera. Implicational (semilinear) logics I: Basic notions and hierarchy. Archive for Mathematical Logic, 49(4):417-446, 2010.

[11] P. Cintula and C. Noguera. A general framework for mathematical fuzzy logic. In P. Cintula, P. Hájek, and C. Noguera, editors, Handbook of Mathematical Fuzzy Logic - Volume 1, volume 37 of Studies in Logic, Mathematical Logic and Foundations. 103-207, London, 2011.

[12] A. Di Nola, F. Esteva, L. Godo, and F. Montagna. Varieties of BL-algebras. Soft Computing, 9(12):875-888, 2005.

[13] F. Esteva, J. Gispert, L. Godo, and F. Montagna. On the standard and rational completeness of some axiomatic extensions of the monoidal t-norm logic. Studia Logica, 71(2):199-226, 2002.

[14] F. Esteva and L. Godo. Monoidal t-norm based logic: Towards a logic for left-continuous t-norms. Fuzzy Sets and Systems, 124(3):271-288, 2001

[15] F. Esteva, L. Godo, and E. Marchioni. Fuzzy logics with enriched language. In P. Cintula, P. Hájek, and C. Noguera, editors, Handbook of Mathematical Fuzzy Logic - Volume 2, volume 38 of Studies in Logic, Mathematical Logic and Foundations. 627-711, London, 2011.

[16] F. Esteva, L. Godo, and C. Noguera. On expansions of WNM t-norm based logics with truth-constants. Fuzzy Sets and Systems, 161(3):347-368, 2010.

[17] F. Esteva, L. Godo, and C. Noguera. Fuzzy logics with truth hedges revisited. In G. M. Sylvie Galichet, Javier Montero, editor, Proc. of the 7th Conference of the European Society of Fuzzy Logic and Technology, EUSFLAT - LFA 2011, pages 146-152, Aix-Les-Bains, France, 2011. Atlantis Press.

[18] O. Fasching. Gödel homomorphisms as Gödel modal operators. To appear in Fundamenta Informaticae, 2012.

[19] P. Grundy. Doing pragmatics. Hodder education, London, 2008.

[20] P. Hájek. Metamathematics of Fuzzy Logic, volume 4 of Trends in Logic. Kluwer, Dordrecht, 1998.

[21] P. Hájek. On very true. Fuzzy Sets and Systems, 124(3):329-333, 2001.

[22] P. Hájek. Some hedges for continuous t-norms logics. Neural Network World, 12(2):159-164, 2002.

[23] P. Hájek and D. Harmancová. A hedge for Gödel fuzzy logic. International Journal of Uncertainty, Fuzziness and Knowledge-Based Systems, 8(4):495-498, 2000.

[24] P. Hájek, F. Montagna, and C. Noguera. Arithmetical complexity of first-order fuzzy logics. In P. Cintula, P. Hájek, and C. Noguera, editors, Handbook of Mathematical Fuzzy Logic - volume 2, number 38 in Studies in Logic, Mathematical Logic and Foundations, chapter XI, pages 853 - 908. College Publications, London, 2011.

[25] P. Hájek and V. Novák. The sorites paradox and fuzzy logic. International Journal of General Systems, 32:373-383, 2003.

[26] R. Horčík, C. Noguera, and M. Petrík. On n-contractive fuzzy logics. Mathematical Logic Quarterly, 53(3):268$288,2007$.

[27] S. Jenei and F. Montagna. A proof of standard completeness for Esteva and Godo's logic MTL. Studia Logica, 70(2):183-192, 2002.

[28] G. Lakoff. Hedges: a study in meaning criteria and the logic of fuzzy concepts. Journal of Philosophical Logic, 2:458-508, 1973.

[29] R. Markkanen and H. Schröder. Hedging: A Challenge for Pragmatics and Discourse Analysis. In R. Markkanen and H. Schröder (Eds.), Hedging and discourse, De Gruyter, Berlin, 1997.

[30] F. Montagna. Storage operators and multiplicative quantifiers in many-valued logics. Journal of Logic and Computation, 14(2):299-322, 2004.

[31] A. A. Monteiro. Algebrés de Heyting symétriques. Portugaliae Mathematica, 39(1-4):1-239, 1980.

[32] V. Novák. A horizon shifting model of linguistic hedges for approximate reasoning. In Proc. 5th IEEE Internat. 
Conf. on Fuzzy Systems, New Orleans, pp. 423-427, 1996.

[33] V. Novák. A comprehensive theory of trichotomous evaluative linguistic expressions. Fuzzy Sets and Systems, 159(22): 2939-2969, 2008.

[34] V. Novák, I. Perfilieva, and J. Močkoř. Mathematical Principles of Fuzzy Logic, Kluwer, Boston/Dordrecht, 1999.

[35] H. Rasiowa. An Algebraic Approach to Non-Classical Logics. North-Holland, Amsterdam, 1974.

[36] U. Straccia and F. Bobillo. Mixed integer programming, general concept inclusions and fuzzy description logics. Mathware and Soft Computing, 14:247-259, 2007.

[37] C. B. Tresp and R. Molitor. A description logic for vague knowledge. In H. Prade, editor, Proceedings XIII ECAI, pages 361-365. John Wiley and Sons, 1998.

[38] V. Vychodil. Truth-depressing hedges and BL-logic. Fuzzy Sets and Systems, 157(15):2074-2090, 2006.

[39] L. A. Zadeh. A fuzzy set theoretic interpretation of linguistic hedges. Journal of Cybernetics, 2(3):4-34, 1972. 